\title{
BMSAP
}

Bulletins et mémoires de la Société d'Anthropologie de Paris

$18(1-2) \mid 2006$

2006(1-2)

\section{Connaissances médicales actuelles, cotation des enthésopathies : nouvelle méthode}

New scoring system of enthesopathies based on present medical data

\section{Sébastien Villotte}

\section{(2) OpenEdition}

1 Journals

\section{Édition électronique}

URL : https://journals.openedition.org/bmsap/1325

DOI : $10.4000 /$ bmsap. 1325

ISSN : 1777-5469

Éditeur

Société d'Anthropologie de Paris

\section{Édition imprimée}

Date de publication : 1 juin 2006

Pagination : 65-85

ISSN : 0037-8984

\section{Référence électronique}

Sébastien Villotte, «Connaissances médicales actuelles, cotation des enthésopathies : nouvelle méthode », Bulletins et mémoires de la Société d'Anthropologie de Paris [En ligne], 18 (1-2) | 2006, mis en ligne le 14 juin 2010, consulté le 01 juin 2021. URL : http://journals.openedition.org/bmsap/1325 DOI : https://doi.org/10.4000/bmsap.1325

\section{cc) (†)}

Les contenus des Bulletins et mémoires de la Société d'Anthropologie de Paris sont mis à disposition selon les termes de la licence Creative Commons Attribution-NonCommercial-NoDerivatives 4.0 International License. 


\title{
CONNAISSANCES MÉDICALES ACTUELLES, COTATION DES ENTHÉSOPATHIES : NOUVELLE MÉTHODE
}

\author{
NEW SCORING SYSTEM OF ENTHESOPATHIES BASED ON PRESENT MEDICAL DATA
}

\author{
Sébastien VILLOTTE ${ }^{1}$
}

RÉSUMÉ

Depuis près de 20 ans, de nombreux travaux ont pour objectif de documenter les comportements des populations du passé à partir des enthésopathies. Cependant, dans ces études, la distinction entre sain et pathologique a été effectuée arbitrairement et l'absence de système d'étude standardisé limite les comparaisons entre les différents travaux. L'objectif de cet article est de proposer une méthode d'étude des enthésopathies fondée sur les données médicales actuelles. Il est possible de distinguer deux types d'enthèses selon la nature du tissu d'insertion - fibreux ou fibrocartilagineux. Les variations d'aspects (normaux ou pathologiques) en fonction de cette distinction permettent d'envisager des systèmes génériques de cotation du remodelage osseux pour 18 sites d'insertions appendiculaires et les insertions rachidiennes des ligaments jaunes. Ces systèmes de cotation en trois stades concernent quatre groupes d'enthèses, définis en fonction de la nature de l'insertion et de l'aspect du remodelage. Les trois premiers groupes comprennent des insertions fibrocartilagineuses. Les traits considérés dans la cotation tentent de correspondre au plus près aux descriptions anatomiques et anatomopathologiques de ces insertions. Le quatrième groupe concerne des insertions fibreuses pour lesquelles les processus de remodelage sont très peu documentés. Avec cette méthode, les erreurs intra- et inter- observateur sont inférieures à $10 \%$. Son application à des squelettes de sujets dont l'âge, le sexe et l'activité pratiquée sont connus permettrait, entre autres, de discuter de la pertinence respective de l'utilisation d'enthèses fibreuses et fibrocartilagineuses dans les interprétations sur les activités pratiquées par les populations du passé.

Mots-clés : enthèses fibrocartilagineuses, enthèses fibreuses, enthésopathies, méthode de cotation.

\section{ABSTRACT}

Over the last twenty years, many studies have used enthesopathies as an indicator of the behaviour of past populations. However, in these works, the distinction between healthy and pathological aspects has been arbitrary. Moreover the lack of standardised observation methods exacerbates this problem and limits the comparison between different studies. The aim of this study is to propose a new method of studying enthesopathies based on present medical data. It is possible to distinguish two types of entheses according to the nature of the tissue of insertion: fibrous or fibrocartilaginous. The variations between normal and pathological aspects based on this distinction enable the proposition of four generic scoring systems. These systems were applied to 18 sites of appendicular insertions and spinal column insertions of yellow ligaments. Under each system, three scoring stages are defined representing different stages of the remodelling process. The first three groups include fibrocartilaginous insertions. We used anatomical and anatomopathological descriptions to define the stages. Such descriptions are rare for the fourth group which concerns

1. UMR 5199, PACEA, Laboratoire d'Anthropologie des Populations du Passé, Université Bordeaux 1, avenue des Facultés, 33405 Talence CEDEX, France, e-mail : s.villotte@anthropologie.u-bordeaux1.fr 
fibrous insertions. With this method, intra-and inter-observer errors are less than 10\%. Application of this scoring method on archaeological skeletons of known age, sex and activity would allow us to discuss the respective relevance of the use of fibrous and fibrocartilaginous entheses for the interpretation of behaviour and activities of past human populations.

Keywords: fibrocartilaginous entheses, fibrous entheses, enthesopathies, scoring system.

\section{INTRODUCTION}

Les activités et les comportements des populations du passé ne sont bien souvent documentés que par les traces matérielles (outils, ossements...). Parmi celles-ci, les restes humains sont utilisés comme source de renseignements sur les activités de ces populations (pour une revue, voir : Capasso et al. 1999). En effet, le squelette humain peut subir des modifications qui ne sont pas attribuables à des désordres systémiques mais qui représentent l'expression de la plasticité de l'os sous la pression de forces extra-corporelles et internes subies au cours de la vie (Kennedy 1989). Ces marques peuvent avoir des origines variées : activités musculaires intenses, postures, traumatismes, contaminations, comportements sanitaires ou alimentaires...

Parmi les différents types de modifications, le remodelage osseux au niveau des enthèses a très souvent été utilisé en anthropologie biologique pour discuter des activités physiques des populations du passé (Dutour 1986 ; Crubézy 1988 ; Hawkey, Merbs 1995 ; Robb 1998). Le terme enthèse semble s'être peu à peu imposé dans la littérature pour désigner les zones d'insertion des muscles, des ligaments et des capsules articulaires au niveau de l'os (Niepel, Sit'aj 1979 ; Resnick, Niwayama 1983 ; Benjamin et al. 2002). La Cava (1959) est le premier à s'être inspiré du mot grec enthesis (= insertion) pour désigner par enthésites les affections liées à l'inflammation des sites d'insertion. Actuellement, le terme enthésopathie, proposé par Niepel et Sit'aj (1979) pour indiquer une modification pathologique liée aux zones d'insertions, sans préciser ni sa nature ni son étiologie, est préféré à celui d'enthésite.

De nombreuses méthodes de cotation de l'aspect osseux des insertions ligamentaires et tendineuses ont été proposées (Crubézy 1988 ; Hawkey 1988 ; Robb 1998 ; Stirland 1998 ; Mariotti et al. 2004). Cependant, comme le soulignent à juste titre Mariotti et al. (2004), il n'existe pas de définition stricte d'une enthésopathie sur le squelette. La principale limite des méthodes existantes réside ainsi dans le choix fait par l'auteur de considérer l'évolution de tel ou tel trait de remodelage comme indicateur de l'évolution d'une intensité lésionnelle, sans utiliser les descriptions anatomiques existantes.

L'objectif de cette article est de proposer une nouvelle méthode de cotation du remodelage osseux au niveau des zones d'insertions, fondée sur la documentation existante concernant l'anatomie et l'anatomopathologie des insertions musculaires et ligamentaires (pour revue, voir : Benjamin, McGonagle 2001 ; Benjamin et al. 2002). Ces travaux permettent, dans un premier temps, de distinguer les insertions suivant leur nature, fibreuse ou fibrocartilagineuse. Il est alors possible d'envisager selon cette distinction leur aspect « sain » sur os sec. Dans un second temps, les données médicales permettent de caractériser, pour les principaux processus lésionnels, le type d'enthèse susceptible d'être atteint et les aspects que pourront prendre ces atteintes sur le squelette. Les traits considérés dans cette méthode ne sont donc pas choisis arbitrairement, comme ils l'étaient par le passé. Au contraire, le choix des critères tente d'être au plus près des descriptions médicales d'enthèses saines et pathologiques, assurant la fiabilité de la méthode. L'application d'une telle méthode pourra alors permettre des comparaisons avec les travaux de médecine du sport et du travail afin de proposer des interprétations rigoureuses sur les activités des populations du passé.

\section{LES DEUX TYPES D'ENTHÈSES : DU VIVANT AUX VESTIGES OSSEUX}

\section{Les enthèses fibrocartilagineuses}

Ces enthèses sont typiques des zones d'ossification secondaire des os longs ; elles se rencontrent aussi sur les os courts des mains et des pieds et sur les vertèbres (Benjamin, Ralphs 1998 ; Benjamin, McGonagle 2001). Quatre zones tissulaires successives peuvent être distinguées pour ces enthèses (Cooper, Misol 1970 ; Benjamin et al. 1986) :

- l'extrémité du tendon ou du ligament ;

- une zone de fibrocartilage non calcifié ; 
- une zone de fibrocartilage calcifié ;

- l'os sous-chondral.

La transition entre les deux premières zones est graduelle. Une limite nette et régulière entre le fibrocartilage non calcifié et calcifié, appelée « tidemark », est observée en microscopie photonique et électronique (Cooper, Misol 1970 ; Benjamin et al. 1986). L'interface entre le fibrocartilage calcifié et l'os souschondral est, quant à elle, constituée d'un système complexe de multiples chevauchements entre les deux tissus (Cooper, Misol 1970 ; Hurov 1986). Les quatre couches sont présentes dans la partie centrale de l'enthèse, où le périoste est absent. À la périphérie, dans la zone la plus éloignée de l'angle formé entre le tendon et l'os, le fibrocartilage est plus rare et les fibres collagènes tendineuses ou ligamentaires s'intriquent avec le périoste (Benjamin et al. 1986).

Sur le squelette, les enthèses fibrocartilagineuses saines sont exemptes de foramen vasculaire et présentent une surface lisse. Benjamin et al. ont proposé une explication à chacune de ces deux caractéristiques. Premièrement, le fibrocartilage forme un « tampon » durant la vie qui empêche la prolifération vasculaire (Benjamin, McGonagle 2001 ; Benjamin et al. 2002). Deuxièmement, c'est la surface de la couche de fibrocartilage calcifié, macroscopiquement lisse et régulière, qui serait observée sur os sec, et non l'os sous-chondral. En effet, le fibrocartilage calcifié se conserve, au moins en partie, après disparition des tissus mous. Benjamin et al. (1986) l'ont montré pour différentes insertions, dans une étude portant sur des cadavres provenant de salles de dissection, alors qu'Henderson et Gallant (2005) ont observé la couche de fibrocartilage calcifié au niveau de l'origine commune des muscles épicondyliens latéraux sur l'humérus de squelettes humains du Moyen Âge.

De nombreuses études médicales décrivent des lésions d'insertions fibrocartilagineuses, notamment grâce aux travaux portant sur les enthésopathies mécaniques et les spondylarthropathies. Les descriptions précises des atteintes du fibrocartilage calcifié et de l'os

\begin{tabular}{|c|c|c|c|}
\hline $\begin{array}{l}\text { Modifications } \\
\text { observées sur le } \\
\text { squelette }\end{array}$ & $\begin{array}{l}\text { Modifications } \\
\text { décrites dans } \\
\text { la littérature }\end{array}$ & $\begin{array}{c}\text { Nature de } \\
\text { l'enthésopathie }\end{array}$ & Sources \\
\hline \multirow{5}{*}{$\begin{array}{c}\text { Excroissance } \\
\text { osseuse ou calcique } \\
=\text { enthésophyte }\end{array}$} & $\begin{array}{l}\text { Dépôt calcique } \\
\text { au niveau de } \\
\text { la « tidemark» }\end{array}$ & Dégénérative (SN) & $\begin{array}{l}\text { Durigon, Paolaggi 1991; Kumagai } \\
\text { et al. } 1994 \text {; Milz et al. } 2004\end{array}$ \\
\hline & \multirow{3}{*}{ Cal de réparation } & Dégénérative (SN) & Milz et al. 2004 \\
\hline & & Dégénérative (MT) & Dupont et al. 1983 \\
\hline & & Inflammatoire (SpA) & Ball 1971 ; Resnick, Niwayama 1983 \\
\hline & $\begin{array}{l}\text { Enthésophyte } \\
\text { d'hyperostose }\end{array}$ & Maladie hyperostosique & Resnick et al. 1975 \\
\hline $\begin{array}{l}\text { Irrégularité de } \\
\text { la surface }\end{array}$ & $\begin{array}{l}\text { Irrégularité de } \\
\text { la « tidemark » }\end{array}$ & Dégénérative (SN) & $\begin{array}{l}\text { Durigon, Paolaggi } 1991 \text {; Kumagai } \\
\text { et al. } 1994 \text {; Milz et al. } 2004\end{array}$ \\
\hline \multirow{3}{*}{ Ostéolyse } & \multirow{3}{*}{ Érosion } & Dégénérative (SN) & Jiang et al. 2002 \\
\hline & & Inflammatoire (SpA) & Ball 1971 ; Resnick, Niwayama 1983 \\
\hline & & Inflammatoire (ECE) & Chan et al. 2004 \\
\hline \multirow{3}{*}{ Foramens } & \multirow{3}{*}{$\begin{array}{l}\text { Vascularisation } \\
\text { accrue de l'enthèse }\end{array}$} & Dégénérative (SN) & Kumagai et al. 1994 ; Milz et al. 2004 \\
\hline & & Dégénérative (MT) & Dupont et al. 1983 ; Potter et al. 1995 \\
\hline & & Inflammatoire (SpA) & Ball 1971 ; D'Agosting, Breban 2003 \\
\hline Géodes & $\begin{array}{l}\text { Arrachements } \\
\text { osseux }\end{array}$ & Dégénérative (MT) & Husson et al. 1991 \\
\hline
\end{tabular}

Tabl. I - Correspondance supposée entre les modifications osseuses observées pour les insertions fibrocartilagineuses et les descriptions lésionnelles, au niveau du fibrocartilage calcifié et de l'os sous-jacent, rencontrées dans la littérature. $S N$ : sénescence ; $M T$ : microtraumatismes ${ }^{2}$; SpA : spondylarthropathies ; ECE : enthésopathie calcifiante érosive.

Table I-Supposed link between the observed osseous modifications of fibrocartilaginous entheses and pathological descriptions of calcified fibrocartilage and underlying bone mentioned in the literature. SN: senescence; MT: microtrauma; SpA: spondyloarthropathy; ECE: calcifying tendinitis with cortical bone erosion.

2. La plupart des auteurs considèrent, à l'heure actuelle, que la majorité des atteintes microtraumatiques des tendons et de leur insertion sont des atteintes dégénératives et non pas inflammatoires (voir : Annaert 2000). 
permettent de mieux appréhender les modifications observables sur le squelette : irrégularité de surface, excroissances osseuses ou calciques appelées enthésophytes, érosions et géodes, foramens. Une correspondance entre ces modifications et celles présentées dans la littérature médicale est proposée dans le tableau I. Lors d'une enthésopathie, la surface de l'enthèse n'est pas toujours uniformément atteinte. Ainsi, les altérations provoquées par une hyper-sollicitation mécanique sont plus importantes et plus nombreuses à la périphérie de l'insertion, là où le fibrocartilage est rare (Nakama et al. sous presse). Aussi, il est intéressant de distinguer le centre et le contour au niveau d'une enthèse fibrocartilagineuse.

\section{Les enthèses fibreuses}

Ces insertions se trouvent dans les régions du squelette appendiculaire où l'épaisseur de la corticale est importante. Elles sont caractéristiques de l'attache des fibres tendineuses au niveau des diaphyses des os longs (Benjamin, Ralphs 1998 ; Benjamin, McGonagle 2001). L'anatomie des enthèses fibreuses est relativement simple, mais peu documentée. L'insertion se fait soit directement dans l'os, soit indirectement via le périoste (Benjamin et al. 2002). Sur le squelette, les insertions périostées ne présentent pas d'aspect particulier, les fibres tendineuses n'ayant pas de lien direct avec l'os. À l'inverse, pour les insertions fibreuses osseuses, la zone d'ancrage se présente sous la forme d'une irrégularité ou d'un relief osseux. La surface d'insertion est souvent rugueuse (Benjamin et al. 2002). Nous n'avons pas trouvé dans la littérature de travaux permettant de corréler l'anatomie de ces enthèses avec leur aspect sur le squelette.

La très grande majorité des enthésopathies décrites concernent des insertions fibrocartilagineuses. Ainsi, dans la liste proposée par Niepel et Sit'aj (1979), sur 39 sites fréquemment atteints, un seul (l'insertion humérale du m. deltoïde) correspond à une enthèse fibreuse attestée. Deux raisons principales peuvent être avancées. Premièrement, les insertions diaphysaires subiraient peu de forces de traction et de compression lors de sollicitations intenses ou répétées de l'appareil locomoteur (Benjamin, Ralphs 1998). Deuxièmement, les enthèses pauvres en fibrocartilage sont rarement atteintes au cours d'une spondylarthropathie (Benjamin, McGonagle 2001). Il existe donc peu de descriptions permettant d'envisager sur os sec l'aspect que prendra une enthésopathie inflammatoire ou mécanique pour ce type d'insertion. L'atteinte d'une insertion périostée correspondra sans doute sur le squelette à une réaction périostée dans la zone d'attache. Concernant les enthèses fibreuses osseuses, la littérature médicale ne décrit précisément des modifications que dans les cas d'une maladie hyperostosique ou d'une enthésopathie calcifiante érosive. Pour la maladie hyperostosique, les modifications ont l'aspect de productions osseuses anormales (Resnick et al. 1975). Une enthésopathie calcifiante érosive correspond à une tendinite calcifiante associée à une érosion de l'os cortical (Chevrot et al. 2003). Assez rares, ces atteintes peuvent, entre autres, être observées au niveau des insertions du $\mathrm{m}$. grand pectoral et du $\mathrm{m}$. deltoïde sur l'humérus, ainsi que pour les insertions du m. grand fessier et du m. grand adducteur sur le fémur (Hayes et al. 1987 ; Hottat et al. 1999 ; Chevrot et al. 2003 ; Flemming et al. 2003). L'érosion peut s'étendre jusqu'à la moelle osseuse ou rester superficielle et ses dimensions apparaissent comme très variables. Elle peut, de plus, être associée à des réactions périostées ou à de petites excroissances osseuses.

\section{MÉTHODE DE COTATION DES ENTHÉSOPATHIES SUR LE SQUELETTE}

\section{Matériel}

La série archéologique utilisée provient de la nécropole antique de la rue Jacques Brel, dans la ville de Saintes (Charente-Maritime, France). La totalité $(\mathrm{N}=76)$ des individus adultes, soit 34 hommes, 23 femmes et 19 sujets de sexe indéterminé, a été observée. Les critères de détermination du sexe sont : la morphologie et la morphométrie de l'os coxal, les mesures extra-pelviennes (Murail et al. 1999). Les critères de détermination de l'âge adulte sont : la fusion de la crête iliaque à l'ilium et la fusion de l'extrémité sternale de la clavicule (Webb, Suchey 1985).

\section{Remarques méthodologiques préliminaires}

Le polymorphisme des marques du remodelage osseux au niveau des enthèses est très important. Deux constats méthodologiques en découlent :

- la prise de mesure est laborieuse et difficilement reproductible (Stirland 1998). Une méthode qualitative d'étude apparaît comme une solution rapide et plus reproductible, malgré la subjectivité d'une cotation ;

- afin d'envisager les différents aspects du remodelage, il est préférable de proposer une méthode 
d'étude en plusieurs stades. Cependant, comme la corrélation entre intensité de remodelage osseux et intensité lésionnelle est ténue, un nombre restreint de stades semble plus rigoureux. Un système de cotation en trois stades a été choisi.

\section{Démarche méthodologique}

\section{Étape 1 : Choix des sites étudiés}

La sélection des sites étudiés a été effectuée selon deux critères :

- les sites devaient présenter une amplitude de remodelage suffisante pour pouvoir être cotés ;

- la variabilité de l'évolution du remodelage ne devait pas être trop grande afin de ne pas empêcher toute tentative d'échelonnage.

D'après ces critères, 18 sites appendiculaires d'insertion (tabl. II) et les insertions rachidiennes des ligaments jaunes ont été sélectionnés.

\section{Étape 2 : Caractérisation des remodelages}

Pour chaque insertion, toute variation par rapport à la « normale » a été étudiée. La localisation de l'atteinte (périphérique ou centrale) au niveau de l'insertion considérée a aussi été relevée. Un schéma d'évolution du remodelage osseux, spécifique à chaque enthèse, a ainsi pu être établi.

\section{Étape 3 : Regroupement des sites}

Quatre processus de remodelage ont pu être distingués, permettant d'isoler quatre groupes d'insertions présentant des remodelages osseux analogues.

Groupe 1 : insertions fibrocartilagineuses appendiculaires dont le remodelage peut concerner le centre ou le contour de l'enthèse. Fréquemment, ces deux parties sont conjointement atteintes. Le remodelage du contour, qui se présente sous la forme de déformation ou d'ostéoformation (enthésophyte), est difficilement mesurable.

Groupe 2 : insertions fibrocartilagineuses appendiculaires dont le remodelage peut concerner le centre ou le contour de l'enthèse. Alors que les atteintes du centre sont inhabituelles, le remodelage du contour, qui se présente sous la forme d'ostéoformation (enthésophyte), est très fréquent et facilement mesurable. L'association d'une atteinte du contour et du centre est exceptionnelle.

Groupe 3 : insertions fibrocartilagineuses rachidiennes pour lesquelles il est impossible de distinguer un centre et un contour. Le remodelage se présente sous la forme d'ostéoformation (enthésophyte) facilement mesurable.

Groupe 4 : insertions diaphysaires considérées comme fibreuses (concernant la nature de ces enthèses, voir la discussion). Il est impossible de distinguer un centre et un contour pour ces insertions. Le schéma de remodelage se caractérise par une irrégularité croissante de la surface. Exceptionnellement, une lacune corticale peut être observée.

\section{Étape 4 : Élaboration de systèmes d'étude}

Pour chaque groupe précédemment défini, un système de cotation est proposé. Ces systèmes de cotation se fondent sur une tentative de compréhension des aspects osseux observés à partir des descriptions trouvées dans la littérature et présentées dans la partie précédente.

Système pour le groupe 1 : deux échelles de remodelage, l'une pour le contour, l'autre pour le centre, ont été réalisées. Elles permettent d'attribuer un score à chacun de ces remodelages. Pour ces insertions, les atteintes concernent fréquemment les deux localisations, les deux scores sont donc additionnés afin de déterminer un stade.

Système pour le groupe 2 : deux échelles de remodelage, l'une pour le contour, l'autre pour le centre, ont été réalisées. Ces deux échelles sont indépendantes. En effet, le remodelage concerne, dans la majorité des cas, uniquement le contour ; de plus, quand une atteinte du centre est observée, elle est quasiment toujours isolée.

Système pour le groupe 3 : une échelle de remodelage fondée sur la dimension de la production osseuse.

Système pour le groupe 4: deux échelles de remodelage, l'une fondée sur l'irrégularité croissante de la zone d'insertion, l'autre sur la dimension de la lacune corticale, ont été réalisées. Ces deux échelles sont indépendantes. En effet, le remodelage se présente, dans la quasi-totalité des cas, sous la forme d'une irrégularité croissante ; de plus, quand une lacune osseuse est observée, elle est quasiment toujours isolée.

\section{Étape 5 : Test de la méthode sur une autre série archéologique}

Afin de vérifier que, pour les insertions considérées, tous les remodelages avaient été envisagés, la méthode (formée des quatre systèmes de cotation) a été appliquée à une série archéologique comprenant 305 individus adultes 
(32 hommes, 202 femmes et 71 sujets de sexe indéterminé). Le matériel ostéologique étudié provient du cimetière du couvent des Sœurs Grises, situé à Beauvais (Oise, France). Les critères de détermination du sexe sont : la morphologie et la morphométrie de l'os coxal, les mesures extra-pelviennes (Murail et al. 1999, 2005). Les critères de détermination de l'âge adulte sont : la fusion de la crête iliaque à l'ilium et la fusion de l'extrémité sternale de la clavicule (Webb, Suchey 1985) et le remodelage de la surface sacro-pelvienne iliaque (Schmitt 2005). À de rares exceptions près (par exemple : modification traumatique exubérante proche d'une zone d'insertion), la méthode de cotation a toujours pu être appliquée. Cette étude a notamment permis de confirmer le caractère inhabituel des lésions au niveau du centre de l'insertion, pour le groupe 2, et la rareté des lacunes corticales pour le groupe 4 .

\section{Systèmes de cotation du remaniement osseux}

Groupe 1 (fig. 1, 2)

Ce groupe comprend six insertions fibrocartilagineuses du squelette appendiculaire (tabl. II). Les modifications observées concernent, soit séparément soit conjointement, le centre et le contour de l'attache.

\begin{tabular}{|c|c|c|c|c|}
\hline Gr. & Muscle(s) & Localisation & Code & Nature \\
\hline 1 & M. subscapulaire & $\begin{array}{l}\text { Humérus : partie supéro-médiale du } \\
\text { tubercule mineur }\end{array}$ & HSC & FC \\
\hline 1 & $\begin{array}{l}\text { MM. supra épineux et infra } \\
\text { épineux (terminaison commune) }\end{array}$ & $\begin{array}{l}\text { Humérus : facettes supérieure et moyenne } \\
\text { du tubercule majeur }\end{array}$ & HSI & FC \\
\hline 1 & $\begin{array}{l}\text { MM. épicondyliens médiaux } \\
\text { (origine commune) }\end{array}$ & $\begin{array}{l}\text { Humérus : face antérieure et médiale de } \\
\text { l'épicondyle médial }\end{array}$ & HEM & FC \\
\hline 1 & $\begin{array}{l}\text { MM. épicondyliens latéraux } \\
\text { (origine commune) }\end{array}$ & $\begin{array}{l}\text { Humérus : face antérieure et latérale de } \\
\text { l'épicondyle latéral }\end{array}$ & HEL & FC \\
\hline 1 & M. biceps brachial & Radius : moitié médiale de la tubérosité & RBB & FC \\
\hline 1 & $\begin{array}{l}\text { MM. semi-menbraneux et biceps } \\
\text { fémoral (origine commune) }\end{array}$ & $\begin{array}{l}\text { Os coxal : face postérieure de la tubérosité } \\
\text { ischiatique }\end{array}$ & CSB & FC \\
\hline 1 & M. petit fessier & Fémur : face antérieure du grand trochanter & FPF & $\mathrm{FC}$ \\
\hline 1 & M. moyen fessier & Fémur : face latérale du grand trochanter & FMF & FC \\
\hline 1 & M. ilio-psoas & Fémur : sommet du petit trochanter & FIP & FC \\
\hline 2 & M. triceps brachial & Ulna : partie postérieure de l'olécrane & UTB & FC \\
\hline 2 & M. quadriceps fémoral & $\begin{array}{l}\text { Patella : moitié antérieure de la base et face } \\
\text { antérieure }\end{array}$ & PQF & FC \\
\hline 2 & M. triceps sural & $\begin{array}{l}\text { Calcaneus : moitié inférieure de la face } \\
\text { postérieure }\end{array}$ & CTS & FC \\
\hline 4 & M. grand pectoral & $\begin{array}{l}\text { Humérus : lèvre antérieure du sillon } \\
\text { intertuberculaire }\end{array}$ & HGP & $\mathrm{F}$ \\
\hline 4 & M. deltoïde & Humérus : tubérosité deltoïdienne & HDE & $\mathrm{F}$ \\
\hline 4 & M. rond pronateur & Radius : partie moyenne de la face latérale & RRP & $\mathrm{F}$ \\
\hline 4 & M. grand fessier & Fémur : tubérosité glutéale & FGF & $\mathrm{F}$ \\
\hline 4 & $\begin{array}{l}\text { MM. vaste médial, grand } \\
\text { adducteur et long adducteur }\end{array}$ & $\begin{array}{l}\text { Fémur : tiers moyen de la lèvre médiale de } \\
\text { la ligne âpre }\end{array}$ & FLA & $\mathrm{F}$ \\
\hline 4 & M. soléaire & Tibia : moitié inférieure de la ligne oblique & TSO & $\mathrm{F}$ \\
\hline
\end{tabular}

Tabl. II - Insertions du squelette appendiculaire considérées, avec le groupe de cotation auxquelles elles appartiennent (Gr.) et la nature supposée de l'insertion, fibrocartilagineuse $(F C)$ ou fibreuse $(F)$.

Table II-Considered insertions on the appendicular skeleton; their scoring group $(G r$. and the supposed nature of the insertion, fibrocartilaginous $(F C)$ or fibrous $(F)$. 


\section{Remaniement du contour}

La modification majoritairement rencontrée au niveau du contour de ces insertions correspond à une production enthésophytique. Trois degrés sont distingués suivant l'ampleur de ce remaniement. Une valeur, indiquée entre parenthèses, est attribuée à chacun de ces degrés :

Absent (0) : contour régulier ;

Mineur (1) : contour saillant ou irrégulier ;

Majeur (2) : enthésophyte(s).

Le tableau III présente, pour les insertions considérées, la partie du contour la plus souvent atteinte (correspondant à la partie de l'insertion la plus éloignée de l'angle tendon/os) ainsi que des remarques sur les spécificités des remaniements.

\section{Remaniement du centre}

Toutes les modifications listées dans le tableau I peuvent être observées au centre de ces enthèses. Les traces de vascularisations sont considérées arbitrairement à partir de trois foramens et seules les géodes dont le diamètre est supérieur à $1 \mathrm{~mm}$ sont prises en compte.
Trois degrés sont distingués suivant l'ampleur du remaniement : absent, mineur ou majeur. Une valeur, indiquée entre parenthèses, est attribuée à chacun de ces degrés.

Absent (0) : surface régulière, absence de foramen ou de géode

\section{Mineur (1) : a) ou b)}

a) Moins de la moitié de la surface de l'insertion présente une des modifications listées dans le tableau I,

b) Toute la surface présente une irrégularité légère, sous forme de petites aspérités.

Majeur (2) : Plus de la moitié de la surface d'insertion présente des modifications importantes. Plusieurs types de remaniements associés sont le plus souvent observés.

\section{Détermination du stade}

L'addition des valeurs attribuées pour le centre et le contour permet de déterminer le stade :

$$
\begin{aligned}
& 0=\text { stade } A \\
& 1-2=\text { stade } B \\
& 3-4=\text { stade } C
\end{aligned}
$$

\begin{tabular}{|c|c|c|c|c|}
\hline Site & $\begin{array}{l}\text { Bord } \\
\text { généralement } \\
\text { atteint }\end{array}$ & $\begin{array}{l}\text { Remaniement } \\
\text { mineur }\end{array}$ & $\begin{array}{l}\text { Remaniement } \\
\text { majeur }\end{array}$ & $\begin{array}{l}\text { Partie du contour dont le remodelage } \\
\text { n'est pas à prendre en compte }\end{array}$ \\
\hline HSI & Latéral & $\begin{array}{l}\text { Contour saillant } \\
\text { ou irrégulier }\end{array}$ & Production amorphe & \\
\hline HSC & Distal et latéral & Idem & Petite crête & \\
\hline HEM & Médial & Idem & Idem & \\
\hline HEL & Latéral & Idem & Idem & \\
\hline RBB & Médial & Idem & $\begin{array}{l}\text { Petite crête ou petits } \\
\text { enthésophytes }\end{array}$ & \\
\hline CSB & Proximal et latéral & $\begin{array}{l}\text { Petit enthésophyte } \\
\text { localisé }\end{array}$ & $\begin{array}{l}\text { Plusieurs enthésophytes } \\
\text { importants }\end{array}$ & $\begin{array}{l}\text { Médiale : insertion du grand ligament } \\
\text { sacro-sciatique }\end{array}$ \\
\hline FIP & Médial & $\begin{array}{l}\text { Contour saillant } \\
\text { ou irrégulier }\end{array}$ & Enthésophytes & $\begin{array}{l}\text { Médio-distale, à la jonction entre petit } \\
\text { trochanter et diaphyse : insertion } \\
\text { fibreuse du muscle iliaque, existence } \\
\text { de nombreuses variations anatomiques }\end{array}$ \\
\hline FPF & Distal et latéral & Idem & Idem & \\
\hline FMF & Tout le contour & Idem & Idem & \\
\hline
\end{tabular}

Tabl. III - Enthèses du groupe 1 : zone du contour préférentiellement atteinte, aspects des remaniements mineurs et majeurs les plus souvent observés. Pour des illustrations de ces remaniements, voir la figure 1.

Table III-Group 1 entheses: the preferentially modified part of the contour and most commonly observed aspects of minor and major modifications. For illustrations of these modifications see figure 1. 


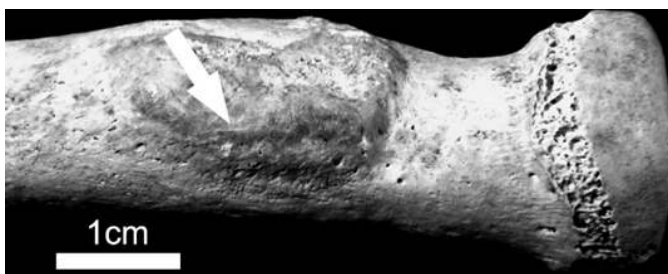

$\mathrm{c}$
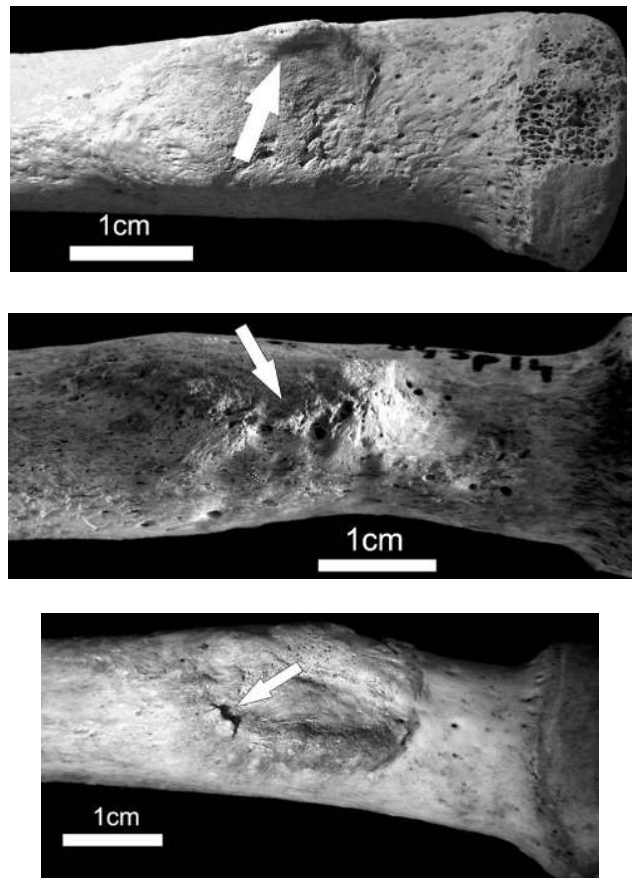

g

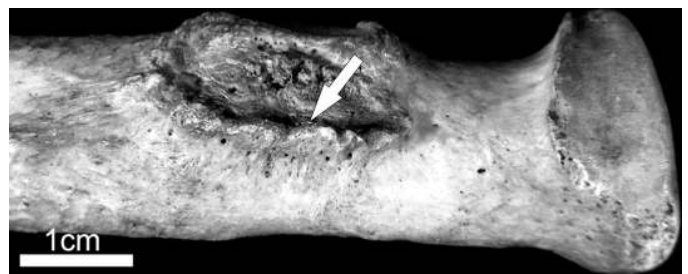

b

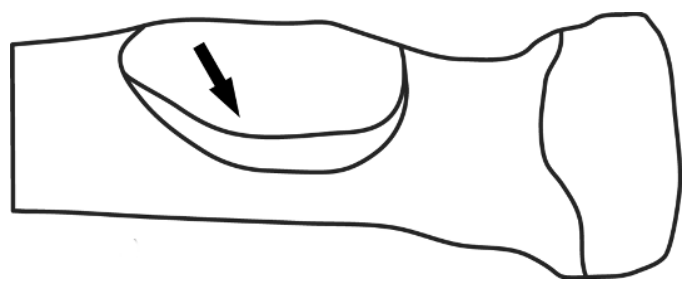

d
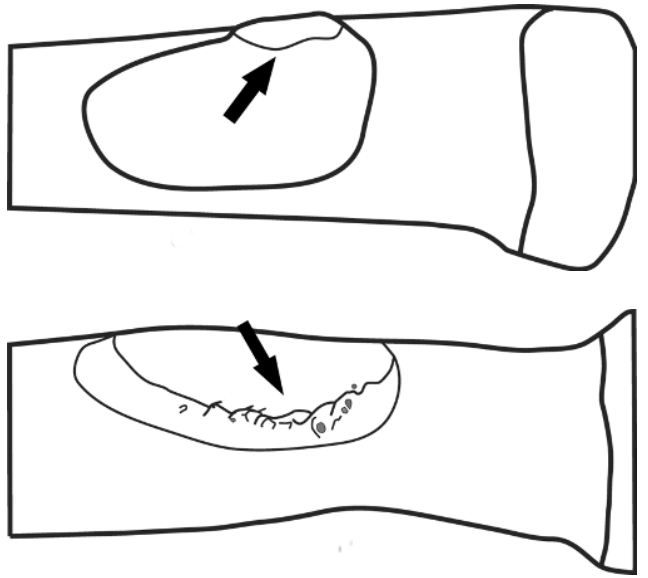

$\mathrm{h}$
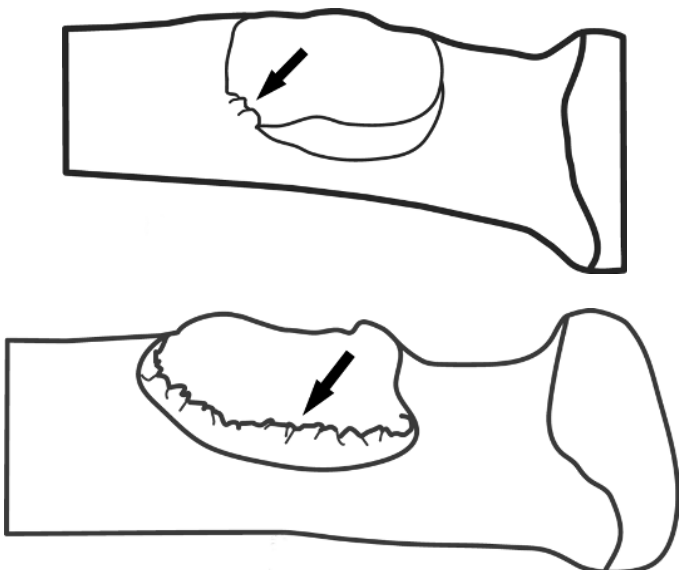

Fig. 1 - Enthèses du groupe 1 : remodelage du contour, exemple de l'insertion du $m$. biceps brachial sur le radius (RBB).

$a$ (cliché) et b (schéma) - Le contour de cette insertion est régulier, sans trace de remodelage (flèche).

Une valeur de 0 est attribuée pour le contour d'une insertion du groupe 1 présentant cet aspect.

$c$ (cliché) et d (schéma) - Le contour de cette insertion forme une petite saillie régulière (flèche).

Une valeur de 1 est attribuée pour le contour d'une insertion du groupe 1 présentant cet aspect.

$e$ (cliché) et f (schéma) - Le contour de cette insertion montre un aspect irrégulier, sans production osseuse

(flèche). Une valeur de 1 est attribuée pour le contour d'une insertion du groupe 1 présentant cet aspect.

$g$ (cliché) et h (schéma) - Le contour de cette insertion montre un enthésophyte bien distinct (flèche).

Une valeur de 2 est attribuée pour le contour d'une insertion du groupe 1 présentant cet aspect.

$i$ (cliché) et $j$ (schéma) - Le contour de cette insertion montre une crête enthésophyte bien distincte (flèche). Une valeur de 2 est attribuée pour le contour d'une insertion du groupe 1 présentant cet aspect.

Fig. 1-Group 1 entheses: contour modification, example of the radial insertion of $\mathrm{m}$. biceps brachialis (RBB).

$a$ (picture) and $b$ (diagram)-The contour of this insertion is regular, without modification (arrow).

$A$ value of $O$ is allotted for contour of a group 1 enthesis with this appearance.

$c$ (picture) and d (diagram)-The contour of this insertion forms a little regular bump (arrow).

A value of 1 is allotted for contour of a group 1 enthesis with this appearance.

$e$ (picture) and $f$ (diagram)-The contour of this insertion shows an irregular appearance, without osseous production (arrow). A value of 1 is allotted for contour of a group 1 enthesis with this appearance.

$g$ (picture) and $h$ (diagram)-An enthesophyte is present on the contour of this insertion (arrow). $A$ value of 2 is allotted for contour of a group 1 enthesis with this appearance.

$i$ (picture) and $j$ (diagram)-The contour of this insertion forms a clear irregular crest (arrow).

A value of 2 is allotted for contour of a group 1 enthesis with this appearance. 


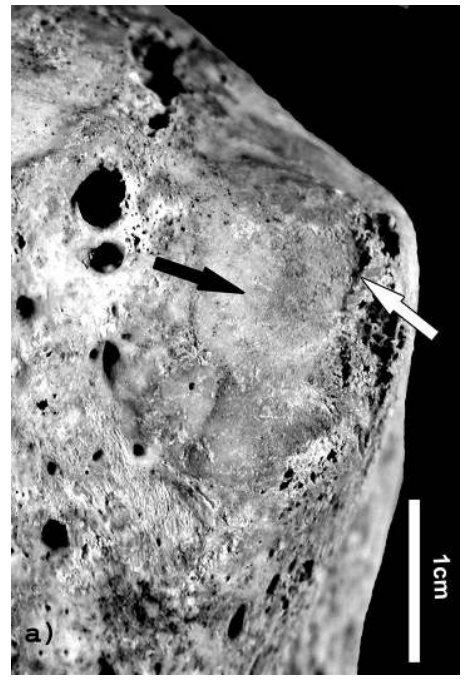

a - Insertion du m. subscapulaire sur l'humérus (HSC). La surface de l'insertion est régulière, sans foramen (flèche noire) : une valeur de 0 est attribuée pour la surface d'une insertion du groupe 1 présentant cet aspect. Le contour de cette insertion est irrégulier (flèche blanche), valeur attribuée : 1. Au final, un stade B est donc attribué pour cette insertion, la somme des valeurs pour le contour et le centre étant comprise entre 1 et 2.

a-Humeral insertion of $\mathrm{m}$. subscapularis (HSC). The surface of this insertion is regular without foramen (black arrow). A value of 0 is allotted for surface of a group 1 enthesis with this appearance. The contour of this insertion is irregular (white arrow), allotted value: 1 . The sum of surface and contour values is included between 1 and 2. therefore a stage $B$ is allotted for this insertion.

$b$ - Insertion des mm. semi-membraneux et biceps fémoral sur l'os coxal (CSB). La surface de l'insertion est régulière mais montre des foramens sur une plage localisée (flèche noire) : une valeur de 1 est attribuée pour la surface d'une insertion du groupe 1 présentant cet aspect. Le contour de cette insertion est régulier (flèche blanche), valeur attribuée : O. Au final, un stade B est donc attribué pour cette insertion, la somme des valeurs pour le contour et le centre étant comprise entre 1 et 2.

$b$-Insertion of $\mathrm{mm}$. semimembranosus and biceps femoris on os coxae (CSB).

The surface of this insertion is regular, showing restricted area with foramina (black arrow). A value of 1 is allotted for surface of a group 1 enthesis with this appearance. The contour of this insertion is regular (white arrow), allotted value: 0 . The sum of surface and contour values is included between 1 and 2, therefore a stage $B$ is allotted for this insertion.
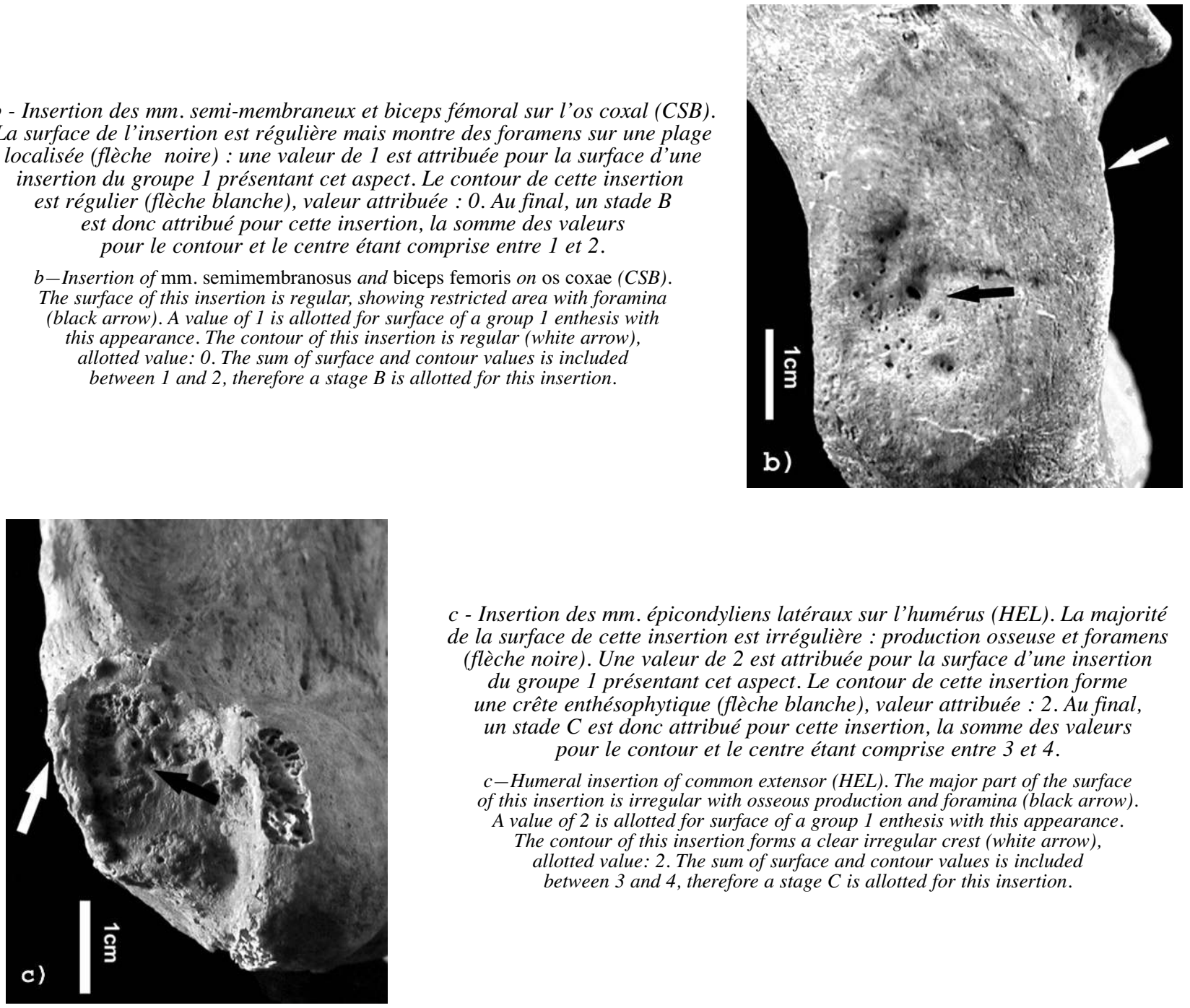

c - Insertion des mm. épicondyliens latéraux sur l'humérus (HEL). La majorité de la surface de cette insertion est irrégulière : production osseuse et foramens (flèche noire). Une valeur de 2 est attribuée pour la surface d'une insertion du groupe 1 présentant cet aspect. Le contour de cette insertion forme une crête enthésophytique (flèche blanche), valeur attribuée : 2. Au final, un stade $C$ est donc attribué pour cette insertion, la somme des valeurs pour le contour et le centre étant comprise entre 3 et 4.

$c-$ Humeral insertion of common extensor (HEL). The major part of the surface of this insertion is irregular with osseous production and foramina (black arrow).

A value of 2 is allotted for surface of a group 1 enthesis with this appearance.

The contour of this insertion forms a clear irregular crest (white arrow),

allotted value: 2 . The sum of surface and contour values is included between 3 and 4 , therefore a stage $C$ is allotted for this insertion.

Fig. 2 - Enthèses du groupe 1. Exemple de remodelage de la surface et d'attribution du stade. Fig. 2-Group 1 entheses. Example of surface modifications and stage allocation. 


\section{Groupe 2 (fig. 3)}

Ce groupe comprend trois insertions fibrocartilagineuses du squelette appendiculaire (tabl. II). Ces insertions présentent, comme celles du groupe précédent, deux zones distinguables : un centre et un contour. Deux schémas de remodelage, indépendants, peuvent être rencontrés. Le plus souvent est observée, à la périphérie de l'enthèse, une production enthésophytique plus ou moins importante. L'attribution du stade dépend de la dimension de cette production osseuse. Le tableau IV indique, pour chaque site, la partie du contour la plus souvent atteinte ainsi que des remarques sur l'aspect des enthésophytes généralement observés. Plus rarement, c'est le centre de l'insertion qui présente un remaniement osseux, sous forme d'une plage d'érosion. L'attribution du stade dépend alors de la dimension de cette plage. Dans les cas exceptionnels où une érosion centrale est associée à une production enthésophytique marginale, le stade de remodelage le plus important est attribué. Ainsi, si par exemple une plage érosive concernant toute la surface

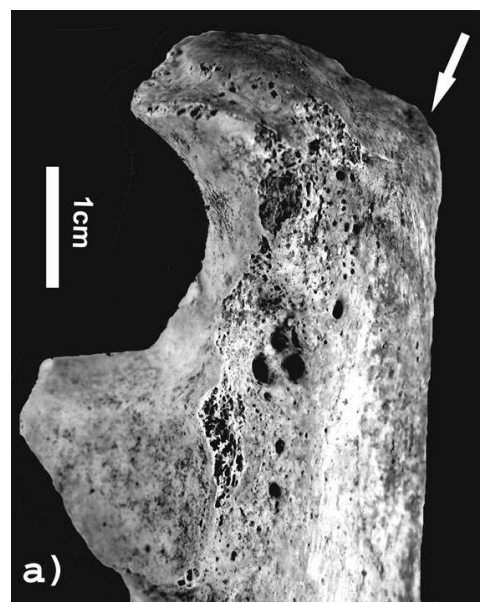

a-Insertion du m. triceps brachial sur l'ulna (UTB). Le contour de l'insertion ne présente pas d'enthésophyte. La surface ne montre pas de trace d'érosion. Un stade A est attribué pour une insertion du groupe 2 ayant cet aspect.

a-Ulnar insertion of $\mathrm{m}$. triceps brachialis (UTB). The contour of this insertion shows no enthesophyte. The surface of he insertion shows no erosion area. A stage A is allotted for a group 2 enthesis with this appearance. (stade C) est associée à un enthésophyte d'un millimètre de longueur (stade $\mathrm{B}$ ), un stade $\mathrm{C}$ est attribué à cette insertion.

Stade A : Absence d'enthésophyte clairement distinct ou de plage d'érosion. Présence possible, au niveau du contour, de petites arêtes perpendiculaires au grand axe de l'insertion.

Stade $B:$ a) ou b)

a) Présence, au niveau de la périphérie, d'un ou de plusieurs petit(s) enthésophyte(s) clairement distinct(s) de longueur inférieure ou égale à $2 \mathrm{~mm}$.

b) Présence, au niveau du centre, d'une plage d'érosion. Sa surface est inférieure ou égale à la moitié de la surface totale d'insertion.

Stade $C:$ a) ou b)

a) Présence, au niveau de la périphérie, d'un ou de plusieurs enthésophyte(s) bien développé(s) de longueur supérieure à $2 \mathrm{~mm}$.

b) Présence, au niveau du centre, d'une plage d'érosion. Sa surface est supérieure à la moitié de la surface totale d'insertion.

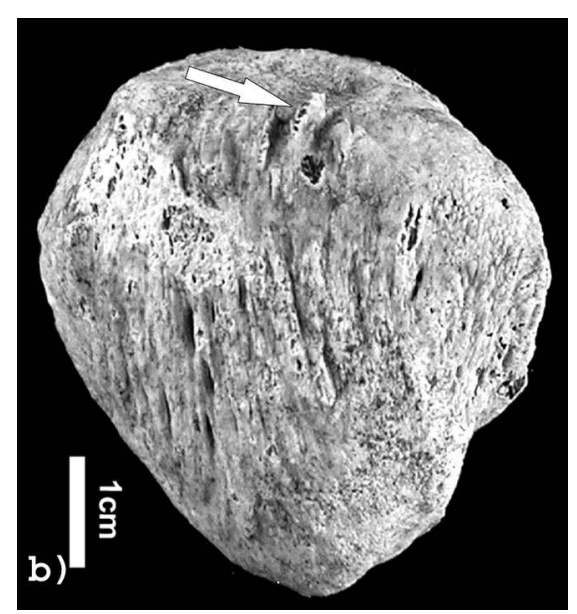

$b$ - Insertion du m. quadriceps fémoral sur la patella $(P Q F)$. Le contour de cette insertion présente un enthésophyte unique $(<2 \mathrm{~mm})$. La surface ne montre pas de trace d'érosion. Un stade B est attribué pour une insertion du groupe 2 ayant cet aspect.

$b$-Patellar insertion of $\mathrm{m}$. quadriceps femoris $(P Q F)$. The contour of this insertion shows a single enthesophyte $(<2 \mathrm{~mm})$. The surface of the insertion shows no erosion area. A stage $B$ is allotted for a group 2 enthesis with this appearance.

Fig. 3a, $b$ - Enthèses du groupe 2 .

Fig. 3a, b-Group 2 entheses. 


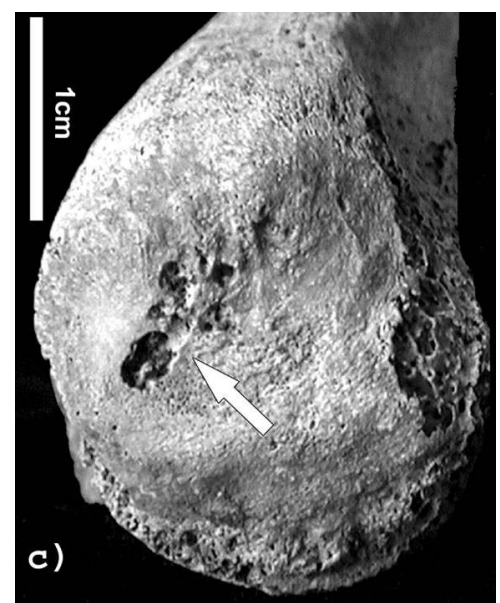

$c$ - Insertion du m. triceps brachial sur l'ulna (UTB).

La surface montre une plage érosive localisée. Un stade B est attribué pour une insertion du groupe 2 présentant cet aspect.

$c$-Ulnar insertion of $\mathrm{m}$. triceps brachialis (UTB).

The surface of this insertion shows a restricted erosion area. A stage $B$ is allotted for a group 2 enthesis with this appearance.

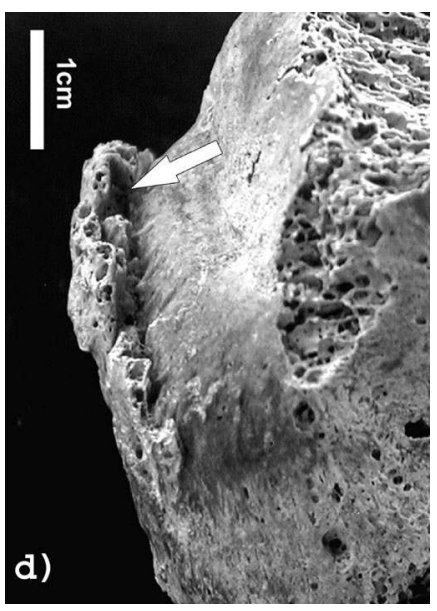

$d$ - Insertion du m. triceps sural sur le calcaneus (CTS). Le contour de cette insertion présente un large enthésophyte (>2 mm). La surface ne montre pas de trace d'érosion. Un stade C est attribué pour une insertion du groupe 2 ayant cet aspect.

$d-$ Calcaneus insertion of $\mathrm{m}$. triceps surae (CTS). The contour of this insertion shows a wide enthesophyte $(>2 \mathrm{~mm})$.

The surface of the insertion shows no erosion area.

A stage $C$ is allotted for a group 2 enthesis with this appearance.

Fig. 3c, $d$ - Enthèses du groupe 2.

Fig. 3c,d-Group 2 entheses.

\begin{tabular}{lccc}
\hline \hline Site & $\begin{array}{c}\text { Bord généralement } \\
\text { atteint }\end{array}$ & Aspect du stade B & Aspect du stade C \\
\hline \hline UTB & Postérieur & $\begin{array}{c}\text { Un enthésophyte à base } \\
\text { étroite ou large }\end{array}$ & Un enthésophyte à base large \\
\hline PQF & Antéro-distal & Quelques enthésophytes & Un «peigne enthésophytique » \\
\hline CTS & Distal & Quelques enthésophytes & Plusieurs larges enthésophytes \\
\hline \hline
\end{tabular}

Tabl. IV - Enthèses du groupe 2 : zone du contour préférentiellement atteinte et aspects $d u$ remodelage majoritairement rencontrés pour les stades $B$ et $C$.

Table IV-Group 2 entheses: The preferentially modified part of the contour and aspects of modification mainly observed in stages $B$ and $C$.

\section{Groupe 3 (fig. 4)}

$\mathrm{Ce}$ groupe est composé des insertions fibrocartilagineuses rachidiennes des ligaments jaunes. Ces ligaments relient les lames vertébrales de l'axis au sacrum. Entre chaque vertèbre se trouvent deux ligaments jaunes qui s'unissent médialement au niveau de l'enthèse. Il n'est pas possible de distinguer pour ces insertions de périphérie et de centre. L'altération typiquement rencontrée est une production enthésophytique (pour revue et discussion, voir Campo Martin et al. 2003). La distinction des stades, modifiée d'après Crubézy (1988), repose sur la taille des enthésophytes. Ces atteintes sont observables au niveau des insertions proximales et distales des ligaments. Chaque insertion proximale et distale est cotée, sans distinction droite/gauche. En effet, cette distinction n'apparaît justifiée, ni d'un point de vue anatomique (union des ligaments au niveau de l'insertion), ni d'un point de vue méthodologique (présence fréquente d'un remodelage central). 


\section{Insertion proximale}

Les altérations peuvent être observées médialement, à la jonction des lames vertébrales ou latéralement, proches des processus articulaires inférieurs.

Stade $A$ : enthésophyte absent ou de longueur inférieure à $1 \mathrm{~mm}^{3}$.

Stade $B$ : présence d'un ou plusieurs enthésophyte(s) de 1 à $4 \mathrm{~mm}$ de long.

Stade $C$ : présence d'un ou plusieurs enthésophyte(s) de plus de $4 \mathrm{~mm}$ de long.

\section{Insertion distale}

Les enthésophytes peuvent être observées médialement, à la jonction des lames vertébrales ou latéralement, sur les lames pouvant alors prendre la forme d'une fine paroi osseuse à la surface ou à la crête irrégulière. Les productions osseuses pourront être particulièrement proches des processus articulaires supérieurs ; il est alors nécessaire de les distinguer des ostéophytes d'arthrose.

Stade $A$ : enthésophyte absent ou de longueur inférieure à $1 \mathrm{~mm}^{3}$. Une fine lame régulière peut être observée, notamment au niveau des thoraciques basses.

\section{Stade B :}

a) Présence d'un ou plusieurs enthésophyte(s) de 1 à $4 \mathrm{~mm}$ de long.

b) Présence d'une lame osseuse à la surface ou à la crête irrégulière de 1 à $4 \mathrm{~mm}$ de largeur.

\section{Stade $C$ :}

a) Présence d'un ou plusieurs enthésophytes de plus de $4 \mathrm{~mm}$ de long.

b) Présence d'une lame à la surface ou à la crête irrégulière de plus de $4 \mathrm{~mm}$ de largeur.

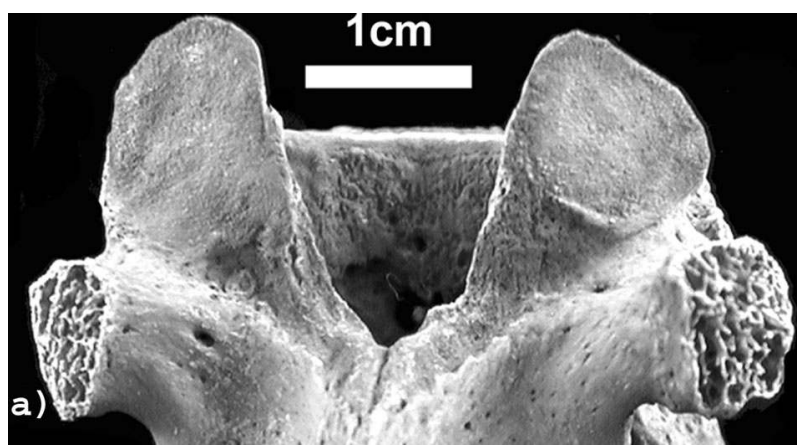

a-Absence d'enthésophyte à la jonction ou sur les lames vertébrales. Un stade A est attribué pour une enthèse du groupe 3 présentant cet aspect.

a-Laminae without enthesophyte. A stage A is allotted for a group 3 enthesis with this appearance.

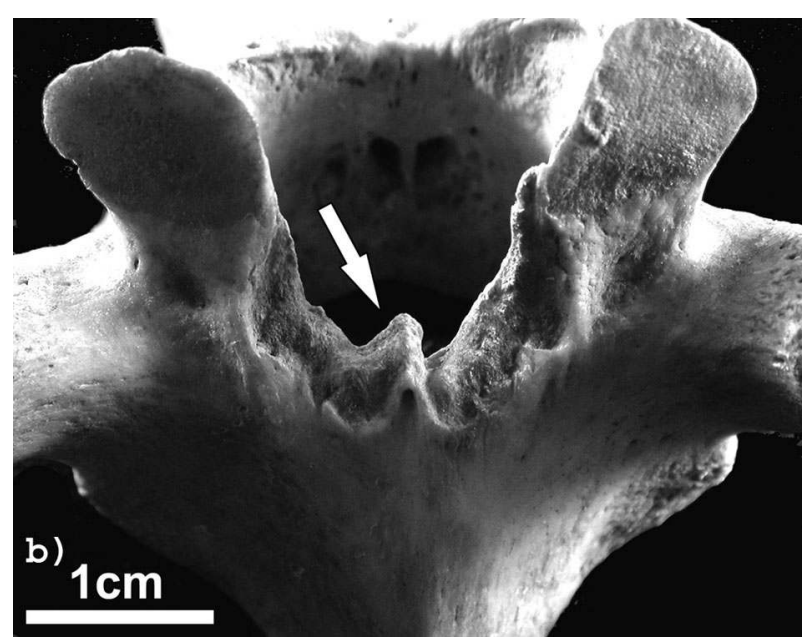

$b$ - Présence d'un enthésophyte $(>1 \mathrm{~mm},<4 \mathrm{~mm})$ à la jonction des lames vertébrales. Un stade B est attribué pour une enthèse du groupe 3 présentant cet aspect.

$b-$ Enthesophyte at the junction of the two laminae
$(>1 \mathrm{~mm},<4 \mathrm{~mm})$. A stage $B$ is allotted for $(>1 \mathrm{~mm},<4 \mathrm{~mm})$. A stage $B$ is allotted for
a group 3 enthesis with this appearance.

Fig. 4a, b-Enthèses du groupe 3, insertions distales.

Fig. 4a, b-Group 3 entheses, distal insertions.

3. Cette limite inférieure permet d'éliminer les variations non pathologiques, toutefois nous ne conseillons pas de l'appliquer pour les vertèbres cervicales. Au niveau de ces vertèbres, les remaniements sont rares (pour la nécropole Jacques Brel : $4 \%$, $\mathrm{N}=8$ ) et clairement distinguables, contrairement aux autres étages. Il apparaît donc justifié de considérer les modifications les plus minimes comme des stades B. 


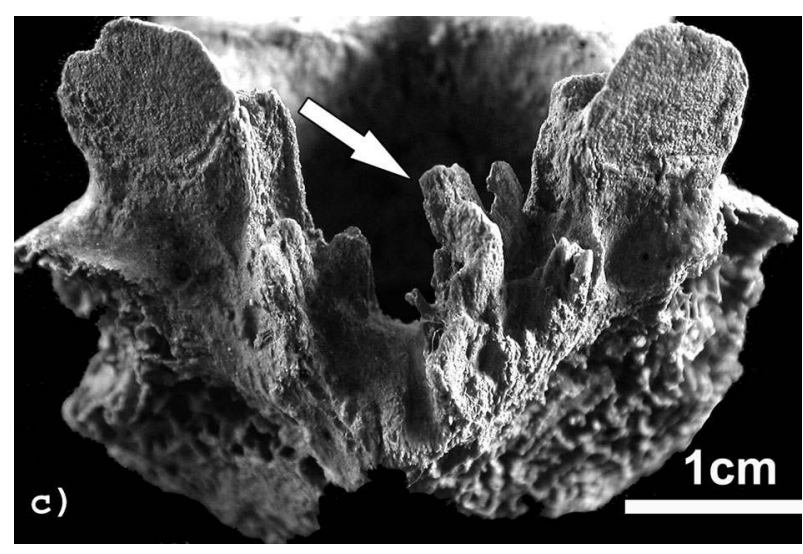

$c$-Présence d'un enthésophyte (> $4 \mathrm{~mm}$ ) au niveau de la lame vertébrale droite. Un stade $C$ est attribué pour une enthèse du groupe 3 présentant cet aspect.

$c-$ Enthesophyte on the right lamina $(>4 \mathrm{~mm})$. A stage $C$ is allotted for a group 3 enthesis with this appearance.

Fig. 4c - Enthèses du groupe 3, insertions distales.

Fig. $4 c-$ Group 3 entheses, distal insertions.

\section{Groupe 4 (fig. 5)}

Ce groupe comporte six enthèses fibreuses (tabl. II). Deux schémas de remodelage peuvent être rencontrés. Le plus courant consiste en une évolution de l'irrégularité de la surface ; il concerne toutes les insertions de ce groupe. Le deuxième type de remodelage, qui n'a été observé que pour l'insertion $\mathrm{du} \mathrm{m}$. grand pectoral, consiste en une absence locale de l'os cortical. La cotation, inspirée de la méthode proposée par Hawkey (1988), se fonde sur le degré d'irrégularité de la surface ou sur la dimension de la lacune corticale. Dans les cas exceptionnels où une absence locale de l'os cortical est associée à une irrégularité de la surface, le stade de remodelage le plus important est attribué. Ainsi, si par exemple une lacune corticale de moins de $2 \mathrm{~cm}$ de long (stade B) est associée à une irrégularité du reste de la surface avec production osseuse (stade C), un stade $\mathrm{C}$ est attribué à cette insertion.
Stade A : surface globalement régulière.

Stade $B:$ a) ou b)

a) Présence d'une irrégularité nette concernant la majeure partie de la zone d'insertion. Absence de production osseuse surélevant des zones de l'insertion.

b) Présence d'une lacune locale de l'os cortical de longueur inférieure à $20 \mathrm{~mm}$.

\section{Stade $C:$ a) ou b)}

a) Présence d'une irrégularité nette concernant la majeure partie de la zone d'insertion. Présence d'un grand ou de plusieurs petits reliefs se distinguant de la zone d'insertion (voir le tableau V pour l'aspect de ces reliefs suivant les sites).

b) Présence d'une lacune locale de l'os cortical de longueur supérieure ou égale à $20 \mathrm{~mm}$.

\begin{tabular}{ll}
\hline \hline \multicolumn{1}{c}{ Site } & \multicolumn{1}{c}{ Aspects des reliefs du stade C } \\
\hline \hline HGP & Petites arêtes perpendiculaires au grand axe de l'insertion ou petits reliefs sans forme caractérisée \\
\hline HDE & $\begin{array}{l}\text { Longues arêtes parallèles au grand axe de l'insertion ou crête unique et irrégulière surélevant toute } \\
\text { l'insertion }\end{array}$ \\
\hline RRP & Petites arêtes disposées en chevron ou crête unique et irrégulière surélevant toute l'insertion \\
\hline FGF & Crête unique, irrégulière et très développée se distinguant de la tubérosité glutéale \\
\hline FLA & Productions osseuses, sans forme caractérisée, se distinguant de la ligne âpre \\
\hline TSO & Crête unique et irrégulière surélevant toute l'insertion \\
\hline \hline
\end{tabular}

Tabl. $V$ - Enthèses du groupe 4 : aspect des remodelages généralement observés pour le stade $C$. Table V-Group 4 entheses: Type of modification generally observed in stage $C$. 


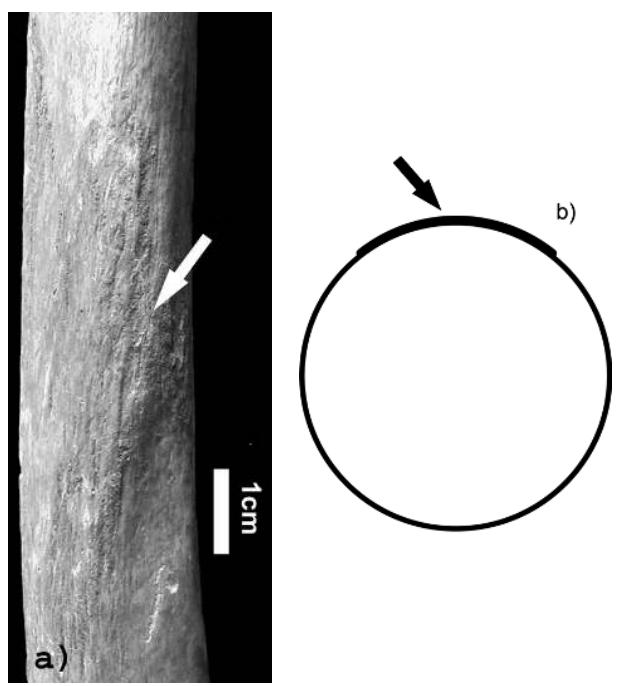

a - Insertion du m. deltoïde sur l'humérus (HDE).

$b$ - Coupe transversale schématique au niveau de cette insertion.

La surface de l'insertion est globalement régulière, sans

aspérité ou relief (flèche). Un stade A est attribué pour une insertion du groupe 4 présentant cet aspect.

$a-$ Humeral insertion of $\mathrm{m}$. deltoïdus (HDE).

$b-$ Schematic cross section of this insertion.

Approximately regular surface, without bump or relief (arrow).

A stage $A$ is allocated for a group 4 enthesis with this appearance.

$c$ - Insertion $d u$ m. rond pronateur sur le radius $(R R P)$.

$d$-Coupe transversale schématique au niveau de cette insertion.

La surface de l'enthèse est irrégulière, mais sans apposition osseuse surélevant l'insertion (flèche). Un stade B est attribué

pour une insertion du groupe 4 présentant cet aspect.

$c$-Radial insertion of $\mathrm{m}$. pronator teres $(R R P)$.

$d$-Schematic cross section of this insertion.

Irregular surface without osseous production (arrow). A stage B is

allocated for a group 4 enthesis with this appearance.
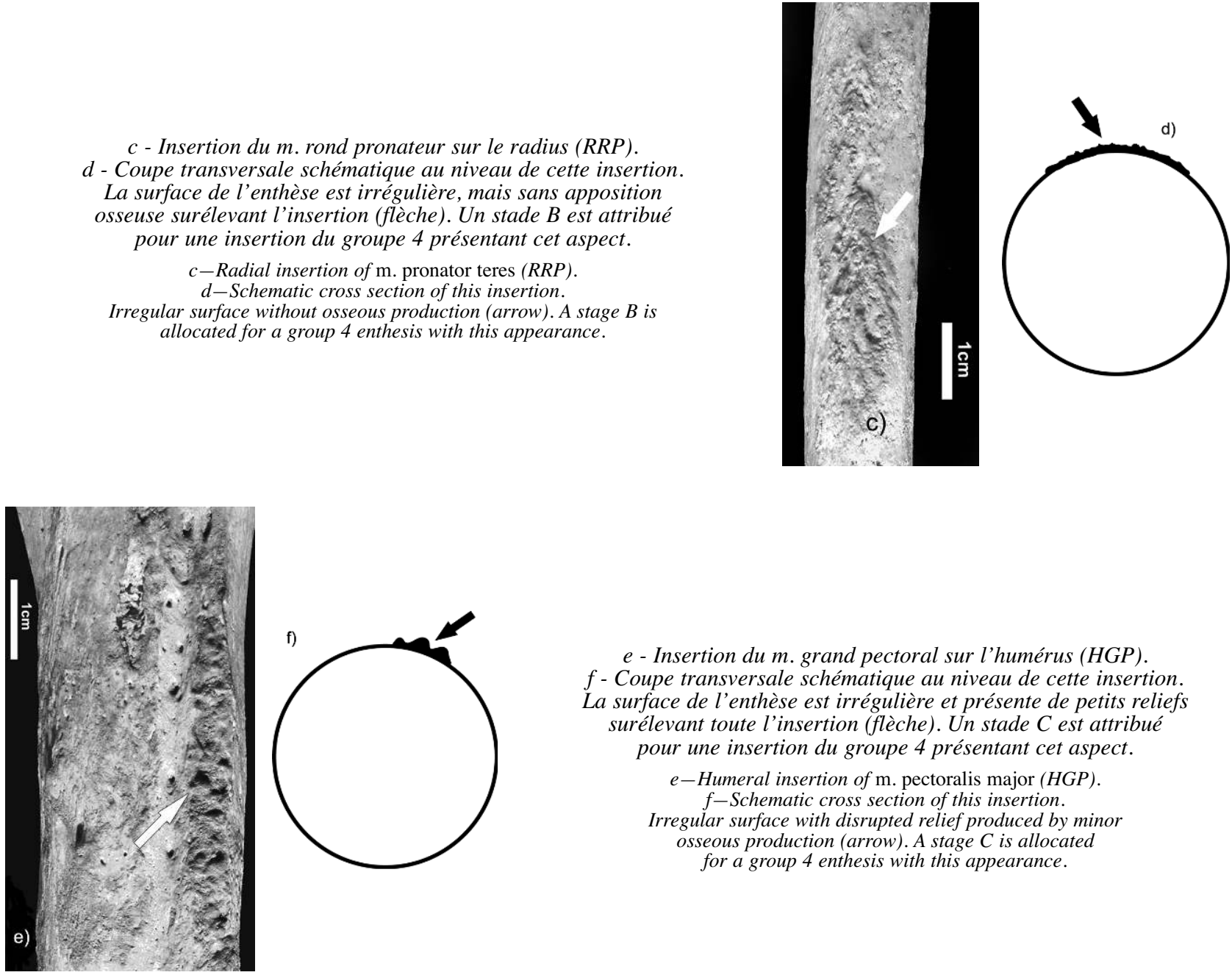

$e$ - Insertion du m. grand pectoral sur l'humérus (HGP).

$f$ - Coupe transversale schématique au niveau de cette insertion. La surface de l'enthèse est irrégulière et présente de petits reliefs surélevant toute l'insertion (flèche). Un stade C est attribué pour une insertion du groupe 4 présentant cet aspect.

$e-$ Humeral insertion of $\mathrm{m}$. pectoralis major $(H G P)$. $f$-Schematic cross section of this insertion.

Irregular surface with disrupted relief produced by minor osseous production (arrow). A stage $C$ is allocated for a group 4 enthesis with this appearance.

Fig. 5a, $b, c, d, e, f$ - Enthèses du groupe 4 .

Fig. 5a, b, $c, d, e, f-$ Group 4 entheses. 


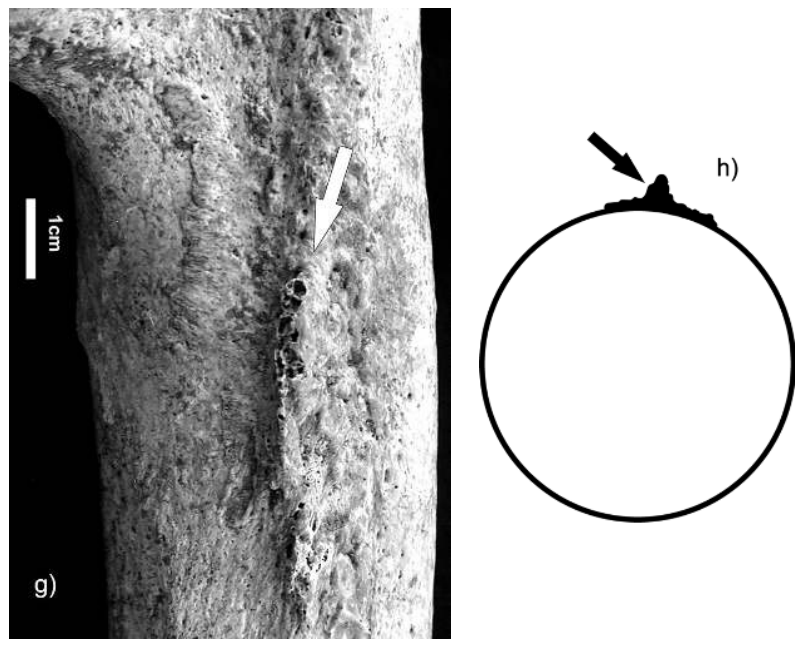

$g$ - Insertion du m. grand fessier sur le fémur (FGF). $h$ - Coupe transversale schématique au niveau de cette insertion. La surface de l'insertion est irrégulière et présente une crête surélevant toute l'insertion (flèche). Un stade C est attribué pour une insertion du groupe 4 présentant cet aspect.

$g-$ Femoral insertion of $\mathrm{m}$. gluteus maximus $(F G F)$. $h$-Schematic cross section of this insertion. Irregular surface with relief caused by a single ridge. A stage $C$ is allocated for a group 4 enthesis with this appearance.

$i$ - Insertion du m. grand pectoral sur l'humérus (HGP). $j$ - Coupe transversale schématique au niveau de cette insertion. Présence d'une lacune corticale (>20 mm), l'os spongieux étant observable (flèche). Un stade C est attribué pour une insertion du groupe 4 présentant cet aspect.

$i-$ Humeral insertion of $\mathrm{m}$. pectoralis major $(H G P)$. $j-$ Schematic cross section of this insertion.

Cortical defect $(>20 \mathrm{~mm}$ ) with cancellous bone observable. A stage $C$ is allocated for a group 4 enthesis with this appearance.
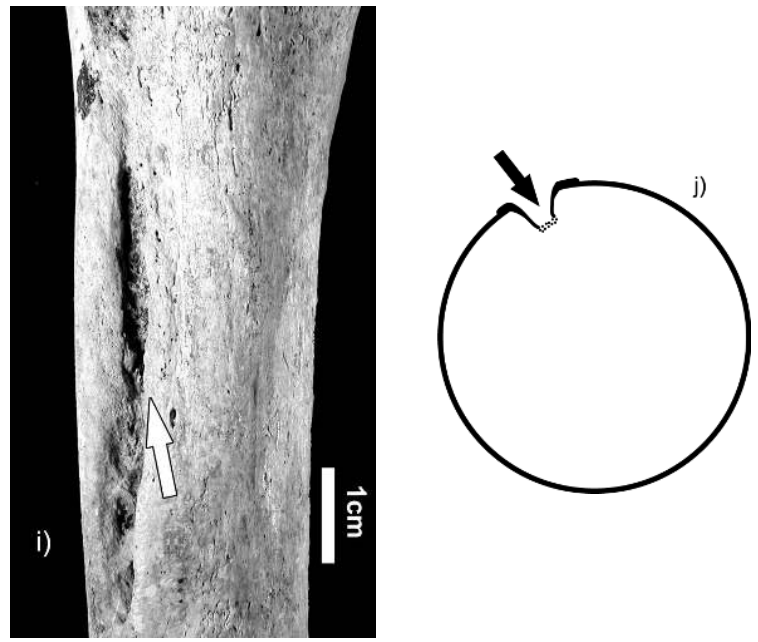

Fig. $5 g, h, i, j$ - Enthèses du groupe 4 .

Fig. 5g, h, i, $j-$ Group 4 entheses.

\section{Reproductibilité de la méthode}

Les tests de reproductibilité ont été effectués sur une série archéologique différente de celle utilisée pour la réalisation de la méthode. Le test intra-observateur a été effectué par le réalisateur de la méthode en deux sessions d'un mois d'intervalle environ. Le test inter-observateur ayant été réalisé après deux semaines d'étude et de discussion autour de cas, les deux personnes peuvent être considérées comme expérimentées. Deux types de calcul ont été effectués à partir des stades attribués par les observateurs. La concordance directe correspond au pourcentage de cotations identiques entre les deux observateurs. Le coefficient de Kappa permet, quant à lui, de juger de la qualité de l'accord entre les deux observateurs. Les résultats des tests sont présentés dans le tableau VI. La reproductibilité de la méthode est globalement bonne avec un pourcentage de concordance supérieur à $90 \%$. Ce résultat est meilleur que celui présenté par Crubézy et al. (2002) mais moins bon que les tests de reproductibilité de la méthode de Mariotti et al., supérieurs à 95 \% (Mariotti et al. 2004). Cependant, l'accord entre les observateurs est considéré comme excellent d'après le coefficient de Kappa. 


\begin{tabular}{|c|c|c|c|c|c|}
\hline & Système & $\mathrm{N}$ cotations & $\begin{array}{c}\text { Concordance } \\
(\%)\end{array}$ & $\begin{array}{l}\text { Coefficient } \\
\text { de Kappa }\end{array}$ & $\begin{array}{l}\text { Accord entre } \\
\text { les observations }\end{array}$ \\
\hline \multirow{5}{*}{ 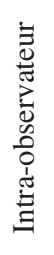 } & 1 & 149 & 95 & 0,89 & Excellent \\
\hline & 2 & 54 & 96 & 0,90 & Excellent \\
\hline & 3 & 179 & 91 & 0,84 & Excellent \\
\hline & 4 & 119 & 89 & 0,79 & Bon \\
\hline & Total & 501 & 92 & 0,86 & Excellent \\
\hline \multirow{5}{*}{ 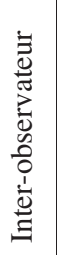 } & 1 & 89 & 85 & 0,75 & Bon \\
\hline & 2 & 48 & 100 & 1 & Parfait \\
\hline & 3 & 151 & 92 & 0,82 & Excellent \\
\hline & 4 & 118 & 89 & 0,62 & Bon \\
\hline & Total & 406 & 90 & 0,83 & Excellent \\
\hline
\end{tabular}

Tabl. VI - Résultats des tests intra- et inter-observateur pour chaque système et pour la méthode : pourcentage de concordance directe entre les observateurs, coefficients de Kappa et jugement de la qualité de l'accord entre les observateurs d'après le coefficient de Kappa.

Table VI-Results of intra- and inter-observer tests for each system and overall result: percentage of direct agreement among observers, the Kappa's coefficients and judgement of the level of agreement among observers according to these coefficients.

\section{DISCUSSION}

\section{Distinction fibrocartilagineux/fibreux}

La distinction entre enthèses fibreuses et fibrocartilagineuses n'est pas exempte de problème. Pour une insertion fibrocartilagineuse, la périphérie ne comporte que peu de fibrocartilage, alors que du fibrocartilage peut être présent en faible quantité au niveau d'une enthèse diaphysaire (Benjamin et al. 1986, 2002). Si de nombreuses études permettent de s'assurer de la nature des insertions des groupes 1, 2 et 3, celle des enthèses du groupe 4 est moins évidente. Les insertions humérales $\mathrm{du} \mathrm{m}$. grand pectoral et $\mathrm{du} \mathrm{m}$. deltoïde, l'insertion $\mathrm{du} \mathrm{m}$. rond pronateur sur le radius ainsi que celle des $\mathrm{mm}$. vaste médial, grand adducteur et long adducteur sur le fémur ont été décrites comme des sites présentant peu ou pas de fibrocartilage (Benjamin et al. 1986, 2002). Cependant, en 2001, Benjamin et McGonagle présentent l'insertion du $\mathrm{m}$. grand pectoral comme une enthèse fibrocartilagineuse (Benjamin, McGonagle 2001). Pour l'insertion du m. grand fessier sur le fémur et celle du $\mathrm{m}$. soléaire sur le tibia, la nature fibreuse de l'insertion est supposée, d'après la localisation diaphysaire de ces enthèses. En dépit de cette incertitude, l'homogénéité du groupe 4 reste très forte du fait des caractéristiques communes des insertions qu'il comprend : localisation diaphysaire, irrégularité fréquente de la surface, schéma de remodelage correspondant en une production osseuse.

\section{Validité de la méthode}

Le système de cotation proposé pour le groupe 1 est, à notre avis, validé par les connaissances médicales actuelles concernant les enthèses fibrocartilagineuses. D'une part, le stade A concorde avec l'aspect d'une insertion non pathologique conservant une fine pellicule de fibrocartilage calcifié après disparition des tissus mous. D'autre part, une correspondance peut être proposée entre les altérations considérées pour attribuer les stades B et C et les différents types d'altérations au niveau de la « tidemark» (et donc observables sur le squelette) décrites dans la littérature. Enfin, le travail de Jiang et al. (2002) démontre l'existence d'une corrélation entre une augmentation de l'importance d'une lésion et une augmentation des modifications osseuses. Concernant les groupes 2 et 3, le stade A semble justifié pour les mêmes raisons. Notons cependant que des enthésophytes sont décrits pour plus de $25 \%$ de sujets sains au niveau de l'insertion du m. triceps sural sur le calcanéus (Resnick, 
Niwayama 1983). La distinction des stades B et C, fondée sur une limite métrique, pose plus de difficultés quant aux interprétations possibles en anthropobiologie. En effet, rien n'atteste la corrélation, quelque peu simpliste, entre une augmentation de longueur d'un enthésophyte et une augmentation de la lésion. De plus, le fait de ne pas avoir observé tous les types de lésions décrits dans la littérature pour ces insertions ne peut pas, à l'heure actuelle, être expliqué. Les aspects osseux observés pour les enthèses réunies dans le groupe 4 sont peu variés ; qualifier une intensité de remodelage est aisé. Cependant, comme nous l'avons déjà souligné, peu de descriptions dans la littérature permettent d'envisager sur os sec l'aspect que prendra une enthèse fibreuse saine ou pathologique. La corrélation entre rugosité et sollicitation musculaire ne repose sur aucun fait documenté. Une extrême prudence quant à leur utilisation dans la discussion d'activités pratiquées par les populations du passé est donc nécessaire.

\section{Les insertions « en fosse »}

La littérature anthropologique fait mention de deux insertions du groupe 4 pouvant prendre un aspect «en fosse » : l'insertion du $\mathrm{m}$. grand pectoral sur l'humérus et $\mathrm{du} \mathrm{m}$. grand fessier sur le fémur. Nous ne discuterons ici que de celle du m. grand pectoral car, alors que la méthode de cotation a été appliquée sur près de 700 squelettes, l'insertion «sous forme de fosse » du m. grand fessier n'a jamais été observée. De nombreuses hypothèses ont été formulées concernant l'insertion « en fosse » du m. grand pectoral sur l'humérus. Considéré comme une variation anatomique (Saunders 1978), comme une lésion due à l'activité (Hawkey 1988 ; Pálfi 1997), voir comme une trace de non-activité (Mafart 1996), cet aspect s'observe parfois avec une fréquence élevée pour les individus immatures et adultes (notamment des jeunes adultes). Parmi ces hypothèses, la plus répandue est celle qui propose une corrélation entre un aspect « en fosse » et une sur-sollicitation musculaire ; elle est d'ailleurs proposée pour d'autres insertions, comme celle du ligament costoclaviculaire sur la clavicule (Hawkey 1988). Cette hypothèse se fonde sur quelques références médicales, notamment la publication d'Husson et collaborateurs (1991), qui présente un mécanisme de microarrachements osseux au niveau d'une enthèse chez de jeunes sportifs. Cependant ce mécanisme a été proposé pour l'insertion fémorale du chef moyen $\mathrm{du}$ $\mathrm{m}$. gastrocnemien et non celle du $\mathrm{m}$. grand pectoral. Plus intéressant est le travail de Fulton et collaborateurs qui décrit, pour cette dernière insertion, des petites lyses osseuses chez les gymnastes, notamment les adeptes des anneaux (1979). Rappelons cependant que la plupart des (rares) descriptions médicales de lacunes corticales humérales sont des cas d'enthésopathies calcifiantes érosives (Chadwick 1989 ; Dürr et al. 1997 ; Flemming et al. 2003) et que ce genre d'atteinte n'est pas clairement corrélé avec une activité physique particulière ou intense. Quoi qu'il en soit, il nous semble possible, et utile, de distinguer deux grands types d'insertion « en fosse », suivant l'aspect du remodelage. Le premier comporte des

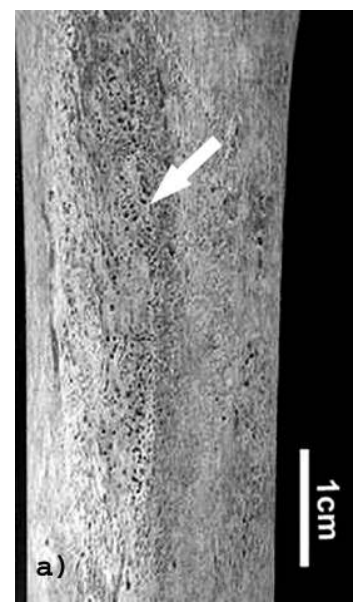

a - Sujet appartenant à la classe d'âge [15-19].

Présence d'une porosité importante au niveau de

l'insertion. Cet aspect est fréquemment rencontré

au niveau des enthèses d'individus immatures.

a-An individual from a [15-19] age group. Marked porosity on the insertion, frequently observed on non adult insertions.

$b$ - Sujet masculin âgé de 21 ans provenant de la " collection de squelettes identifiés du Muséum d'Anthropologie de l'Université de Coimbra». Présence d'une dépression au niveau de l'insertion, associée à une porosité importante. $b-A 21$ years old man from the "Identified Skeletal Collection of the Anthropological Museum of the University of Coimbra". Presence of a depression on the insertion, associated with marked porosity.

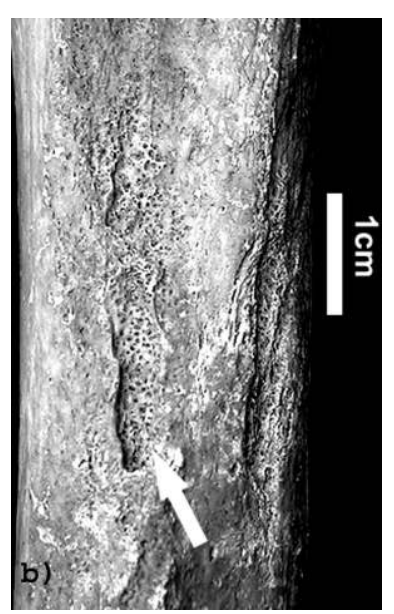

Fig. 6 - Aspects immatures de l'insertion du m. grand pectoral sur l'humérus (HGP).

Fig. 6-Appearance of non adults insertion of $\mathrm{m}$. pectoralis major on the humerus (HGP). 
insertions présentant des traits fréquemment rencontrés pour les insertions d'individus juvéniles (fig. 6a) : porosité, stries, petites aspérités de surface. Notons d'ailleurs que de nombreuses zones d'insertions de sujets immatures sont en fait concaves par rapport aux diaphyses. Les insertions «en fosse » du $\mathrm{m}$. grand pectoral sur l'humérus présentant cet aspect se rencontrent chez des individus non adultes ou chez des très jeunes adultes (moins de 25 ans) (fig. 6b). Ce type de remodelage, pour une insertion proche de la surface métaphysaire, semble être associé aux processus de remodelage et de migration des insertions lors de la croissance osseuse (Hoyte, Enlow 1966 ; Dörfl 1980). Les insertions « en fosse » du m. grand pectoral présentant cet aspect (de même que toutes les enthèses de morphologie « immature ») doivent à notre avis être exclues d'une étude traitant des activités pratiquées. Le deuxième groupe d'insertion « en fosse » se rencontre exclusivement chez les adultes. La fosse apparaît comme une réelle lacune de l'os cortical, de dimension variable, le plus souvent délimitée par des bords réguliers (fig. $5 i$ ).

\section{Les cas où la cotation est impossible : altérations pathologiques ou taphonomiques}

Il existe de nombreux cas où une insertion ne peut être cotée. Les situations sont variées : altérations pathologiques « recouvrant» la zone d'insertion (par exemple : modifications osseuses causées par la tuberculose ou réaction périostée importante), persistance de tissu fibreux, os absent, desquamation superficielle de la corticale au niveau de l'insertion, traces laissées par des racines... Les différentes tentatives que nous avons effectuées pour proposer des critères d'exclusion se sont, jusqu'à présent, révélées insatisfaisantes. Notons que Mariotti et al. (2004) proposent des critères d'exclusion qui, s'ils nous paraissent incomplets et encore trop subjectifs (notamment la notion de doute quant à la nature de l'altération : taphonomique ou pathologique), représentent une bonne piste de travail.

\section{CONCLUSIONS}

De nombreuses réserves ont été formulées face à l'engouement (légitime) de la communauté scientifique devant le potentiel interprétatif des marques de remodelage osseux au niveau des enthèses (Dutour 1992 ; Robb 1998 ; Stirland 1998). Cependant, le choix des traits considérés et l'hypothèse d'une corrélation entre l'évolution du remodelage et l'intensité de l'atteinte n'ont jamais réellement été mis en question. Il est pourtant possible de profiter des nombreuses avancées faites en anatomie et en anatomopathologie pour proposer une méthode d'étude cohérente. Ainsi, les systèmes de cotation pour les enthèses fibrocartilagineuses se fondent sur la mise en évidence de la persistance de la couche de fibrocartilage calcifié après disparition des tissus mous. Cette mise en évidence permet de mieux caractériser l'aspect sain et pathologique de ces insertions, le remodelage observé au niveau d'enthèses fibreuses restant, quant à lui, énigmatique. La définition de critères simples, le faible nombre de stades et l'iconographie proposée en font une méthode reproductible, rapide et facile à appliquer. La principale originalité de cette méthode est de distinguer différents groupes d'enthèses suivant leur nature et le type de remodelage observé. La distinction enthèses fibrocartilagineuses/enthèses fibreuses semble nécessaire pour l'exploitation des données récoltées. En effet, les atteintes mécaniques des enthèses fibrocartilagineuses sont bien connues contrairement à celles des enthèses fibreuses. Cependant, comme des remodelages osseux sont observés au niveau des enthèses fibreuses, les deux catégories sont considérées en anthropobiologie sans distinction pour discuter des activités des populations du passé. Il semble pertinent de documenter les aspects que peuvent prendre les enthèses en fonction des différents facteurs susceptibles de causer des enthésopathies et, pour un même individu, de comparer l'intensité des atteintes selon la nature de l'insertion. Ainsi, une des perspectives possible est l'application de cette méthode à des squelettes de sujets dont l'âge, le sexe et l'activité pratiquée sont connus. Une telle étude permettrait :

- de proposer un modèle d'évolution du remodelage au cours de la vie pour une intensité d'activité donnée ;

- de discuter de la pertinence respective des enthèses fibreuses et fibrocartilagineuses pour des interprétations sur les activités pratiquées ;

- de construire une base de données de référence.

Les cotations attribuées pour des squelettes « non identifiés » pourront alors être comparées à cette base de données afin de proposer des hypothèses sur l'intensité des activités et la répartition des taches pour ces périodes.

\section{Remerciements}

Je tiens à remercier P. Murail, D. Castex et P. Courtaud ainsi que le Service Archéologique de la ville 
de Beauvais, pour la permission d'étudier les séries ostéologiques utilisées dans cette méthode. Merci à D. Henry-Gambier, D. Castex et S. Kacki pour la relecture du texte, ainsi qu'aux deux lecteurs anonymes pour les conseils concernant la clarté de la présentation de la méthode. Ce travail intègre le volet méthodologique d'une thèse financée par le Ministère de la Recherche.

\section{BIBLIOGRAPHIE}

AnNAert (J.-M.) 2000, « Ne dites plus jamais tendinite », Revue de la Médecine Générale 172 : 146-150.

BALL (J.) 1971, Enthesopathy of rheumatoid and ankylosing spondylitis, Annals of the Rheumatic Diseases 30: 213-223.

Benjamin (M.), Evans (E.J.), CopP (L.) 1986, The histology of tendon attachments to bone in man, Journal of Anatomy 149: 89-100.

Benjamin (M.), Kumai (T.), Milz (S.), BoszczyK (B.M.), Boszczyk (A.A.), RALPHS (J.R.) 2002, The skeletal attachment of tendons-tendon "entheses", Comparative Biochemistry and Physiology, Part A 133: 931-945.

Benjamin (M.), McGonagle (D.) 2001, The anatomical basis for disease localisation in seronegative spondyloarthopathy at entheses and related sites, Journal of Anatomy 199: 503-526.

BENJAMin (M.), RAlPHS (J.R.) 1998, Fibrocartilage in tendons and ligaments -an adaptation to compressive load, Journal of Anatomy 193, 4: 481-494.

Campo Martin (M.), González Martin (A.), Rodriguez GonZÁLEZ (A.I.) 2003, El problema de la calcificatión del ligamento amarillo, Propuesta methodológica para su estudio en paleopatologia, in J.A. Sánchez Sánchez (ed.), Actas del V Congreso Nacional de Paleopatología, p. 54-64, http://www.ucm.es/info/aep/congreso.htm

Capasso (L.), Kennedy (K.A.R.), Wilczack (C.A.) 1999, Atlas of occupational markers on human remains, Edigrafital S.p.A., Teramo, 183 p.
Chadwick (C.J.) 1989, Tendinitis of the pectoralis major insertion whith humeral lesions, The Journal of Bone and Joint Surgery [Br] 71-B: 916-918.

Chan (R.), Kim (D.H.), Millett (P.J.), Weissman (B.N.) 2004, Calcifying tendinitis of the rotator cuff with cortical bone erosion, Skeletal Radiology 33: 596-599.

Chevrot (A.), Drape (J.-L.), Moutounet (L.), Godefroy (D.), Dupont (A.-M.) 2003, Enthésopathies calcifiantes érosives, in H. Bard, A. Cotten, J. Rodineau, G. Saillant, J.-J. Railhac (éds), Tendons et enthèses, Sauramps médical, Montpellier, p. 81-92.

CoOPer (R.R.), Misol (S.) 1970, Tendon and ligament insertion, A light and electron microscopic study, The Journal of Bone and Joint Surgery 52-A, 1: 1-20.

CRUBÉZY (E.) 1988, Interactions entre facteurs bio-culturels, pathologie et caractères discrets, Exemple d'une population médiévale : Canac (Aveyron), Thèse de doctorat de Médecine, Université de Montpellier, 417 p.

Crubézy (E.), Goulet (J.), Bruzek (J.), Jelinek (J.), RougÉ (D.), LudES (F.) 2002, Épidémiologie de l'arthrose et des enthésopathies dans une population européenne d'il y a 7700 ans, Revue du Rhumatisme (Éd.fr.) 69 : 1217-1225.

D'Agosting (M.-A.), Breban (M.) 2003, Besoin d'imagerie dans le diagnostic et le suivi des spondylarthropathies, in H. Bard, A. Cotten, J. Rodineau, G. Saillant, J.-J. Railhac (éds), Tendons et enthèses, Sauramps Médical, Montpellier, p. 123-128. 
DöRFL (J.) 1980, Migration of tendinous insertions, I. Cause and mechanism, Journal of Anatomy 131, 1: 179-195.

Dupont (M.), Pasteels (J.-L.), Duchateau (M.), SZPALSKY (M.) 1983, Tendinites corporéales et ostéotendinites, essai de définition des lésions et de leur traitement chirurgical, Acta Orthopaedica Belgica 49, $1-2: 30-41$.

Durigon (M.), PaolagGi (J.-B.) 1991, Enthèse au cours de la vie, in L. Simon, C. Hérisson, J. Rodineau (éds), Pathologie des insertions et enthésopathies, Masson, Paris, p. 12-17.

DürR (H.R.), Lienemann (A.), Silbernagl (H.,) Nerlich (A.), REFIOR (H.J.) 1997, Acute calcific tendinitis of the pectoralis major insertion associated with cortical bone erosion, European Radiology 7: 1215-1217.

Dutour (O.) 1986, Enthesopathies (lesions of muscular insertions) as indicators of the activities of Neolithic Saharan populations, American Journal of Physical Anthropology 71: 221-224.

Dutour (O.) 1992, Activités physiques et squelette humain: le difficile passage de l'actuel au fossile, Bulletins et Mémoires de la Société d'Anthropologie de Paris, n.s., 4 : 233-241.

Flemming (D.J.), Murphey (M.D.), SheKitKa (K.M.), Temple (H.T.), JelineK (J.J.), Kransdorf (M.J.) 2003, Osseous involvement in calcific tendinitis, American Journal of Roentgenology 181: 965-972.

Fulton (M.N.), Albright (J.P.), El-Khoury (G.Y.) 1979, Cortical desmoid-like lesion of the proximal humerus and its occurence in gymnasts (ringman's shoulder lesion), The American Journal of Sports Medicine 7, 1: 57-61.

HAWKEY (D.E.) 1988, Use of upper extremety enthesopathies to indicate habitual activity patterns, $\mathrm{PhD}$ thesis, Arizona State University, 86 p.

Hawkey (D.E.), Merbs (C.F.) 1995, Activity-induces musculoskeletal stress markers (MSM) and subsistence strategy changes among ancient Hudson Bay eskimos, International Journal of Osteoarchaeology 5: 324-338.

Hayes (C.W.), Rosenthal (D.I.), Plata (M.J.), Hudson (T.M.) 1987, Calcific tendinitis in unusual sites associated with cortical bone erosion, American Journal of Roentgenology 149: $967-970$

Henderson (C.Y.), Gallant (A.J.) 2005, A simple method of characterising the surface of entheses, Poster, Paleopathology Association, 32nd Annual North America Meeting, Milwaukee, Wisconsin, April 5th and 6th 2005.
Hottat (N.), Fumière (E.), Delcour (C.) 1999, Calcific tendinitis of the gluteus maximus tendon: CT findings, European Radiology 9: 1104-1106.

Hoyte (D.A.N.), EnLow (D.H.) 1966, Wolff's law and the problem of muscle attachment on resorptive surface of bone, American Journal of Physical Anthropology 24: 205-214.

HUROV (J.R.) 1986, Soft-tissue interface: how do attachments of muscles, tendons, and ligaments change during growth? A light microscopic study, Journal of Morphology 189 , 3: $313-325$.

Husson (J.-L.), Chauveaux (D.), Rochcongar (P.), LANCIEN (G.) 1991, Physiopathologie et anatomo-pathologie des enthésopathies d'insertion par sollicitations mécaniques du système tendino-musculaire, in L. Simon, C. Hérisson, J. Rodineau (éds), Pathologies des insertions et enthésopathies, Masson, Paris, p. 156-166.

Jiang (Y.), Zhao (J.), Van Holsbeeck (M.T.), Flynn (M.J.), OUYANG (X.), GENANT (H.K.) 2002, Trabecular microstructure and surface changes in the greater tuberosity in the rotator cuff tears, Skeletal Radiology 31: 522-528.

KenNEDY (K.A.R.) 1989, Skeletal markers of occupational stress, in M.Y. Iscan, K.A.R. Kennedy (eds), Reconstruction of life from the skeleton, Liss, New York, p. 130-160.

Kumagai (J.), Sarkar (K.), Uhthoff (H.K.) 1994, The collagen types in the attachment zone of rotator cuff tendons in the elderly: An immunohistochemical study, Journal of Rheumatology 21, 11: 2096-2100.

La CAVA (G.) 1959, L'enthésite ou maladie des insertions, La Presse Médicale 67: 9.

MAFART (B.-Y.) 1996, Quelques particularités anthropologiques et paléopathologiques d'une population de moniales médiévales de l'abbaye de Saint-Pierre de l'Almanarre (XIII ${ }^{\mathrm{e}}$-XIV ${ }^{\mathrm{e}}$ siècles, Hyères, Var), in L. Buchet (éd.), L'identité des populations archéologiques, Actes des $X V I^{e}$ rencontres internationales d'archéologie et d'histoire d'Antibes, Éditions APDCA, Sophia-Antipolis, p. 271-286.

Mariotti (V.), Facchini (F.), Belcastro (M.G.) 2004, Enthesopathies-Proposal of a standardized scoring method and applications, Collegium Anthropologicum 28, 1: 145-159.

Milz (S.), Tischer (T.), Buettner (A.), Schieker (M.), Maier (M.), Redman (S.), Emery (P.), McGonagle (D), Benjamin (M.) 2004, Molecular composition and pathology of entheses on the medial and lateral epicondyles of the 
humerus: a structural basis for epicondylitis, Annals of the Rheumatic Diseases 63, 9: 1015-1021.

Murail (P.), BruzeK (J.), BragA (J.) 1999, A new approach to sexual diagnosis in past populations, Practical adjustements from Van Vark's procedure, International Journal of Osteoarchaeology 9: 39-53.

Murail (P.), BruzeK (J.), HouËt (F.), Cunha (E.) 2005, DSP: a tool for probabilistic sex diagnosis using worldwide variability in hip-bone measurements, Bulletins et Mémoires de la Société d'Anthropologie de Paris, n.s., 17, 3-4: 167-176.

Nakama (L.H.), King (K.B.), Abrahamsson (S.), REMPEL (D.M.) sous presse, Evidence of tendon microtears due to cyclical loading in an in vivo tendinopathy model, Journal of Orthopaedic Research.

NIEPEL (G.A.), SiT'AJ (S.) 1979, Enthesopathy, Clinics in Rheumatic Diseases 5, 3: 857-872.

PÁlfi (G.) 1997, Maladies dans l'Antiquité et au Moyen-Âge : paléopathologie comparée des anciens Gallo-Romains et Hongrois, Bulletins et Mémoires de la Société d'Anthropologie de Paris, n.s., 9, 1-2, 205 p.

Potter (H.G.), Hannafin (J.A.), Marwessel (R.M.), DiCarlo (E.F.), O’Brien (S.J.), Altcheck (D.W.) 1995 Lateral epicondilitis: correlation of MR imaging, surgical and histopathologic findings, Radiology 196: 43-46.
RESNICK (D.), NIWAYAMA (G.) 1983, Entheses and enthesopathy, Radiology 146: 1-9.

ReSnick (D.), Shaul (S.), Robins (J.) 1975, Diffuse idiopathic skeletal hyperostosis (DISH): Forestier's disease with extraspinal manifestations, Radiology 115: 513-524.

RoвB (J.E.) 1998, The interpretation of skeletal muscle sites: a statistical approach, International Journal of Osteoarchaeology 8: 363-377.

SAUNDERS (S.R.) 1978, The development and distribution of discontinuous morphological variation of the human infracranial skeleton, Archaeological survey of Canada, Paper no.81, Ottawa, National Museums of Canada, 549 p.

SCHMitT (A.) 2005, Une nouvelle méthode pour estimer l'âge au décès des adultes à partir de la surface sacro-pelvienne iliaque, Bulletins et Mémoires de la Société d'Anthropologie de Paris, n.s., 17, 1-2 : 89-101.

STIRLAND (A.J.). 1998, Musculoskeletal evidence for activity: problems of evaluation, International Journal of Osteoarchaeology 8, 5: 354-362.

WebB (P.A.), SuCHEY (J.M.) 1985, Epiphyseal union of the anterior iliac crest and the medial clavicle in a modern multiracial sample of American males and females, American Journal of Physical Anthropology 68: 457-466. 